\title{
The Nature of Radio Emission from Distant Galaxies: The 1.4 GHz Observations
}

\author{
E. A. Richards \\ National Radio Astronomy Observatory
}

Received —

\footnotetext{
${ }^{1}$ The National Radio Astronomy Observatory is a facility of the National Science Foundation operated under cooperative agreement by Associated Universities, Inc.
} 


\begin{abstract}
We have conducted a deep radio survey with the Very Large Array at 1.4 GHz of a region containing the Hubble Deep Field. This survey overlaps previous observations at $8.5 \mathrm{GHz}$ allowing us to investigate the radio spectral properties of microjansky sources to flux densities greater than $40 \mu \mathrm{Jy}$ at 1.4 $\mathrm{GHz}$ and greater than $8 \mu \mathrm{Jy}$ at $8.5 \mathrm{GHz}$. A total of 371 sources have been catalogued at $1.4 \mathrm{GHz}$ as part of a complete sample within $20^{\prime}$ of the HDF. The differential source count for this region is only marginally sub-Euclidean and is given by $n(S)=(8.3 \pm 0.4) S^{-2.4 \pm 0.1} \mathrm{sr}^{-1} \mathrm{Jy}^{-1}$. Above about $100 \mu \mathrm{Jy}$ the radio source count is systematically lower in the HDF as compared to other fields. We conclude that there is clustering in our radio sample on size scales of $1^{\prime}-40^{\prime}$.

The 1.4 GHz selected sample shows that the radio spectral indices are preferentially steep $\left(\bar{\alpha}_{1.4}=0.85\right)$ and the sources are moderately extended with average angular size $\theta=1.8^{\prime \prime}$. Optical identification with disk-type systems at $z \sim 0.5-0.8$ suggests that synchrotron emission, produced by supernovae remnants, is powering the radio emission in the majority of sources. The 8.5 $\mathrm{GHz}$ sample contains primarily moderately flat spectrum sources $\left(\bar{\alpha}_{8.5}=0.35\right)$, with less than $15 \%$ inverted. We argue that we may be observing an increased fraction of optically thin bremsstrahlung over synchrotron radiation in these distant star-forming galaxies.
\end{abstract}

Subject headings: galaxies: evolution - galaxies: active - galaxies: starburst cosmology: observations - radio continuum: galaxies 


\section{Introduction}

Deep radio surveys show a surface density of radio objects approaching $60 \mathrm{arcmin}^{-2}$ down to $1 \mu \mathrm{Jy}$ (as inferred from fluctuation analyses on the most sensitive VLA images at 1.4 and $8.4 \mathrm{GHz}$ ), or equal to the surface density of $\mathrm{B}=27$ magnitude galaxies (Fomalont et al. 1991, Windhorst et al. 1993, Richards 1996, Richards et al. 1998, hereinafter Paper I). Despite their modest average luminosity of $\mathrm{L} \simeq 10^{22.5} \mathrm{~W} / \mathrm{Hz}$, the sheer number of microjansky sources implies that they dominate the radio luminosity budget of the universe at centimeter wavelengths. However, surprisingly little is known about the physical origin of these objects or their nature.

Some generalizations from studies involving deep 5 and $8.5 \mathrm{GHz}$ VLA fields imaged with HST and ground-based telescopes are possible (Hammer et al. 1995, Windhorst et al. 1995, Fomalont et al. 1997, Richards et al. 1998). At least half of microjansky sources are associated with morphologically peculiar, merging and/or interacting galaxies with evidence for active star-formation (blue colors, infra-red excess, HII-like optical spectra). The remaining identifications are composed of low-luminosity FR Is, ellipticals, Seyferts, LINERs, and luminous star-forming field spirals. Thus a variety of physical mechanisms may be driving the observed evolution among microjansky radio sources, including non-thermal radiation from AGN activity, synchrotron emission associated with diffuse supernova remnants, and thermal emission from HII regions.

Another clue is available from the observed distribution of radio spectral indices. While at millijansky levels, the average spectral index for radio sources is about $\alpha \sim 0.8$ (Donnelly et al. 1987), microjansky sources selected at high frequencies $(\nu \geq 5 \mathrm{GHz})$ have a surprisingly flat spectra of $\alpha=0.3 \pm 0.2$ (Fomalont et al. 1991, Windhorst et al. 1993). Several explanations for this observed flattening compared with sources selected at higher flux density levels are possible, including, free-free absorption, an increasing number 
of synchrotron self-absorbed AGN among the microjansky population, and/or a rising component of thermal radiation from active star-formation.

The purpose of this study is to enlarge the faint radio sample and investigate the radio spectral and morphological properties for a statistically significant sample of microjansky sources. In addition, examination of the optical properties of the identifications may shed insight into the nature of the radio emission in these sources. To further this goal we have observed a region of the sky at $1.4 \mathrm{GHz}$ and $8.5 \mathrm{GHz}$ centered on the Hubble Deep Field, where excellent wide field optical data are available. In Paper I (Richards et al. 1998) we presented the optical identifications for a complete sample of twenty-nine $8.5 \mathrm{GHz}$ selected radio sources in our radio survey of the Hubble Deep Field. The principal conclusion is that the majority ( $\sim 70 \%$ ) of the identifications are with relatively bright ( $\mathrm{R} \sim 22$ mag.) disk systems, many at moderate redshifts $(z=0.4-1)$. In this paper (Paper II) we present the 1.4 GHz observations. In section two we describe our observations and data reduction techniques. Section three presents our source list, while in section four we calculate the spectral index distribution. In $\S 5$ we discuss the spatial clustering of sources in our catalog. Finally, in $\S 6$ we summarize our findings and give conclusions.

\section{The 1.4 GHz Radio Observations and Data Reduction}

In November 1996, we observed a field centered on the Hubble Deep Field ( $\alpha=$ $12^{h} 36^{m} 49.4^{s}$ and $\delta=62^{\circ} 12^{\prime} 58.00^{\prime \prime}(\mathrm{J} 2000)$ ) for a total of 50 hours at $20 \mathrm{~cm}$ in the A-configuration of the VLA. In order to minimize chromatic aberration, we observed in a pseudo-continuum, spectral line mode with $7 \times 3.125 \mathrm{MHz}$ channels centered on intermediate frequencies $1365 \mathrm{MHz}$ and $1435 \mathrm{MHz}$, frequency windows known to be relatively free of radio frequency interference. Each frequency channel was composed of two independent circular polarizations. All knowledge of linear-polarized intensities was lost 
due to the limited number of VLA correlator channels. Visibility data were recorded every $3.3 \mathrm{sec}$ from the correlator.

We monitored the point source $1313+675\left(\mathrm{~S}_{1.4}=2.40 \mathrm{Jy}\right)$, located 6.5 degrees from the HDF, every 40 minutes to provide amplitude, phase, and bandpass calibration. Daily observations of 3C286 with assumed flux densities of $14.91 \mathrm{Jy}$ at $1385 \mathrm{MHz}$ and $14.62 \mathrm{Jy}$ at $1435 \mathrm{MHz}$ provided the absolute flux density scale.

The calibrator observations allowed us to identify baselines with systematic phase or amplitude errors. A few baselines were found to have recurrent amplitude and/or phase closure errors greater than five percent and/or five degrees. All data associated with these suspicious baselines were discarded. To remove bursts of radio frequency interference, we excised all visibilities with flux densities greater than about $10 \sigma$ above the expected rms value of $0.08 \mathrm{Jy}$. This amounted to about $2 \%$ of the data.

After time averaging the $(u, v)$ data from 3.3 to $13 \mathrm{sec}$, we made preliminary $10^{\prime \prime}$ resolution maps which cover the field out to the first sidelobe of the primary beam about $0.8^{\circ}$ from the phase center. We searched these images for bright, confusing sources whose sidelobes might contaminate the noise properties of the inner portion of field. All sources above $0.5 \mathrm{mJy}$ were catalogued.

We then imaged and heavily CLEANed these sources using the full unweighted $(u, v)$ data set. Because the primary beam response changes significantly over our $44 \mathrm{MHz}$ bandpass (about 3\%), it was necessary to deconvolve each of the confusing sources using each $3.125 \mathrm{MHz}$ channel. In addition, the confusing sources were independently deconvolved in each of the circular polarizations (right and left) to account for the 'beam squint' of the VLA antenna. Their CLEAN components were Fourier transformed and then subtracted from the visibility data. Using these "strong source subtracted data sets", we then imaged the inner few arcmin of the field. With this procedure the rms noise was found to be about 
$50 \%$ higher than expected from receiver noise alone. In particular, a few sidelobes from particularly strong sources $(\mathrm{S}>10 \mathrm{mJy})$ located near the half power point and first null of the primary beam were still apparent.

By examining the images made from 30 minute segments of the data, we isolated a few time intervals where the visibility data appeared to be corrupted, possibily due to low level interference. For any of these 30 minute snap-shot images with a a rms noise greater than $50 \%$ of the mean value, the corresponding visibility data were deleted from the final analysis. These data amounted to about seven hours of time; thus, in all, about 42 hours of of high quality data were used to construct the final images. The final combined data set has a rms noise of $7.5 \mu \mathrm{Jy}$, whereas we expected a noise closer to $5 \mu \mathrm{Jy}$.

\subsection{Construction of the $1.4 \mathrm{GHz}$ Images}

Our goal is to map the $40^{\prime}$ field of the $1.4 \mathrm{GHz}$ observations out to the $20 \%$ response of the VLA antennas. There are two complications which make this a difficult task.

First is the sky curvature. While in practice the VLA is often treated as a two dimensional array, in reality the instrumental response to radio emission from the sky is a three dimensional complex function. For observations of short duration, small fields of view, or low resolution, most sources can be adequately deconvolved without reference to the so called 3-D effect. However, the isoplanic assumption fails for sources located further than $\theta \simeq 1 / n_{\text {syn }}$ radians from the phase tracking center, where $n_{\text {syn }}$ is the phase center distance in synthesized beam widths. For the A-array at $1.4 \mathrm{GHz}$ this corresponds to a patch size of about 18 arcmin across.

Thus we chose to approximate the one degree primary beam of our observations by using a number of independent and equidistant facet images. Each facet is constructed from 
the Fourier transform of the data which has been phase shifted to its tangent point on the celestial sphere. In all, 16 facets were used. This technique is known as polyhedral imaging and is discussed at length by Cornwell \& Perley (1992).

One further complication comes from the practical limitation of the extensive computations needed to reduce these these wide field observations. Our entire calibrated and edited data set consists of over $10^{7}$ discrete complex visibilities (after $13 \mathrm{sec}$ time averaging) which must be directly Fourier transformed to compute the sky brightness distribution over approximately the same number of pixels. Because we are interested in radio emission over the entire primary beam, we must properly deconvolve sources within the entire field of view. Although only sources fainter than $0.5 \mathrm{mJy}$ remain in the visibility data after the subtraction of the confusing sources, their collective sidelobes can limit the dynamic range of the final images.

The most accurate method for deconvolution of the faint sources is to CLEAN each of the 16 images in parallel, subtracting each source's sidelobe contribution from all the image facets simultaneously. In this manner, one would recover the sky brightness function free from sidelobe contamination over the entire primary beam. However, in practice, this is a prohibitive computational task. Therefore we opted to CLEAN each of the 16 facets in series using the much more efficient Clark-Hogbom algorithm (Clark 1980). The price paid is that sidelobes from the multitude of sources less than $0.5 \mathrm{mJy}$ are only properly removed locally, within the individual image facet which contains each source.

In order to examine what effect this might have on the rms noise near the center of the image, we performed a simple test. First we made an image of the inner seven arcmin of the field center, heavily CLEANed using the Clark-Hogbom method. Then we made a similar image twice the size of the former, again heavily CLEANed. Comparison of the two images, one relatively free of sidelobe contamination from the far field sources with $\mathrm{S} \leq 0.5$ 
mJy, the other not, yields a first order approximation of the unCLEANed sidelobes left in the center of the field due to our deconvolution method outlined above. About $0.8 \mu \mathrm{Jy}$ of flux density remains per independent beam, and thus increases the noise about $10 \%$ over the thermal noise.

We produced the final image by CLEANing each of the sixteen $2048 \times 2048$ pixel fields $\left(0.4^{\prime \prime}\right.$ pixels) with 10,000 iterations and a 10\% loop gain. This produced an image with a natural resolution of $2.0^{\prime \prime} \times 1.8^{\prime \prime}$ and $\mathrm{P} . \mathrm{A} .=-86^{\circ}$. The rms noise near the center of the field was $7.5 \mu \mathrm{Jy}$, approximately $30 \%$ higher than the value expected from thermal noise alone based on our system temperature (37 K on average), integration time, and bandwidth. The noise in each of the 16 images was found to be fairly uniform, although it increased to as much as $10 \mu \mathrm{Jy}$ in regions near the brightest millijansky sources.

Residual ringlike structure from unCLEANable sidelobes around these strong sources was evident, suggesting that our images are dynamic range limited at about 5000:1. This is typical of blank field deep imaging where the data cannot be effectively self-calibrated. We clipped out regions immediately around these sources, as well as a larger region around one particularly strong source $(\mathrm{S}=35 \mathrm{mJy})$ located at the half-power point of the primary beam. We attribute the dynamic range limitation in this image to pointing fluctuations, which are typically $15^{\prime \prime} \mathrm{rms}$ for the VLA. This effect causes amplitude fluctuations in the apparent brightness of the radio sources, which are of order $1 \%$ at the half power point, and hence induces unCLEANable sidelobes into the image.

Examination of the stronger deconvolved sources in the field with $S_{p}>5$ mJy showed evidence of a radial sidelobe oriented towards the phase tracking center, or center of our images. These sidelobes appeared to be symmetric about these stronger millijansky sources in the field and with first order amplitude of about 10\%. Their width was approximately that of the delay beam. 
These sidelobes were also apparent during our test observations of $1400+621$, and only appear around the off axis observations. Other observations taken at the VLA have not shown similar artifacts and our suspicion is that the problem was an online system recording error with the $3.3 \mathrm{sec}$ visibility data that we chose to use. Examination of VLA data of the same test field taken in an identical observing mode, except with 10 sec visibility integration, showed no sign of the radial sidelobes.

The cause of these artifacts is unknown. They do not appear to be present around the near field, weaker sources (less than about $1 \mathrm{mJy}$ ). As a test of whether these sidelobes might adversely effect our measurements of the flux densities of the stronger millijansky sources, we examined the off-axis flux density measurements of $1400+621$ in images with and without the presence of the radial sidelobes. The measurements agree to within the standard flux density errors. It is more difficult to assess if these radial sidelobes adversely affect the general rms noise in the final $1.4 \mathrm{GHz}$ images.

\section{The Complete Source List}

In order to minimize the introduction of spurious radio sources into our sample, we examined the distribution of the negative pixels in the images to estimate its completeness limit. In general most regions appeared to reflect well behaved Gaussian noise with the most negative peak being about five times the local rms noise. However, one region with a local rms of $7.5 \mu \mathrm{Jy}$ contained an unresolved negative feature with $S_{p}=-63 \mu \mathrm{Jy}$. This feature appeared to be quite isolated and located several arcmin from any of the brighter millijansky confusing sources. No other strange artifacts (e.g., rings, streaks, residual sidelobes) were apparent in the vicinity of this negative 'source'; hence its presence remains

enigmatic. The next most negative pixel value of $-40 \mu \mathrm{Jy}$ is located near a strong confusing source. We therefore adopt $40 \mu \mathrm{Jy}$ to be the formal completeness limit over our entire one 
degree field. Even if there are positive counterparts to the $-63 \mu \mathrm{Jy}$ 'source', there should be no more than a few if the negative and positive pixel amplitude distribution are fairly symmetric about zero. However, we note that the probability of finding a $-9 \sigma$ source within our field is much less than one percent, and demonstrates that the noise properties of this image are not entirely Gaussian.

Next we searched our images for all pixels with $\mathrm{S} \geq 40 \mu \mathrm{Jy}$ and fit these sources with elliptical Gaussians to determine their peak flux densities and positions using the automated AIPS task SAD. In all 314 sources were found within 20 arcmin of the center of the image (20\% power contour).

\subsection{Determination of Discrete Source Angular Sizes and Flux Densities}

Gaussian fitting routines such as SAD are subject to noise dependent biases which cause significant overestimation of source sizes and flux densities (Windhorst et al. 1984, Condon 1997). In order to estimate the effects of population and noise bias in our images, we performed a series of Monte Carlo simulations. Our basic technique was to inject a number of point sources (100) of known flux density into the CLEANed sky images of this field. Then these sources were recovered from the images using SAD and their measured peak and integrated flux densities compared to the input model. We used the ratio $S_{p} / S_{i}$ to determine if the simulated source was significantly broadened by population and/or noise bias.

Because our goal is to set a resolution criteria in the presence of these fitting biases, we performed this simulation as a function of flux density, or alternatively, signal to noise. By examining the distribution of $S_{p} / S_{i}$ at different flux densities, we obtained some idea about at what level we could confidently believe that a given source was resolved in our real 
sky images. For a source of intrinsic dimension $\theta_{\text {maj }} \times \theta_{\text {min }}$ resolved by a beam of extent $a_{m a j} \times a_{m i n}$, the integrated and peak flux densities are related by $S_{i}=S_{p}\left(1+\frac{\theta_{m a j} \times \theta_{\min }}{a_{m a j} \times a_{m i n}}\right)$, thus allowing us to relate the ratio $S_{p} / S_{i}$ directly to a source angular size. By examining the distribution of modeled $S_{p} / S_{i}$ to input $S_{p} / S_{i}$, we were able to adopt a $95 \%$ confidence in the resolution criteria as a function of signal to noise. The resulting best fit data yields $S_{p} / S_{i}(95 \%)<1-\frac{2.3}{\sigma_{s n r}}$ where $\sigma_{s n r}$ is the signal to noise ratio of the radio source. Thus at the detection limit of our images $(40 \mu \mathrm{Jy})$, a source must have a $S_{p} / S_{i}$ value less than 0.57 to satisfy our resolution criterion, or an angular size greater than about $2.7^{\prime \prime}$. It follows that we can resolve only those sources stronger than $70 \mu \mathrm{Jy}$ integrated flux in our complete sample.

Next, we used these same simulations with the sources of angular size that we could have just detected at each signal to noise ratio (e.g., $2.7^{\prime \prime}$ at $S_{p}=40 \mu \mathrm{Jy}$ ). Comparison of the input to recovered peak and integrated flux densities, and angular sizes yields an estimate of the bias induced by Gaussian fitting techniques in the presence of population and noise bias. We corrected our real sky source parameters to account for these biases. In general the peak flux densities of the fitting routines were in good agreement with the models, while the integrated flux densities and angular sizes from the Gaussian fits were overestimated by up to a factor of 1.5 .

As a check on the integrated flux densities for apparently resolved sources in our complete sample obtained from the Gaussian fitting algorithms, we examined the distribution of peak flux densities as measured in various resolution images. Images were constructed at $1^{\prime \prime}, 3.5^{\prime \prime}$, and $6^{\prime \prime}$ resolution in addition to our nominal $2^{\prime \prime}$ naturally weighted image. Table 1 gives the parameters of each image. For those sources which satisfied our initial resolution criterion, we checked that the peak flux density of the source increased with decreasing resolution consistent with the fitted angular size. Those sources which did 
not were considered unresolved and their adopted peak and integrated flux densities were measured on the the $2^{\prime \prime}$ image. Figure 1 shows a greyscale of the inner $10^{\prime}$ of the $1.4 \mathrm{GHz}$ image. In Figure 2, we show the greyscale of the $8.5 \mathrm{GHz}$ mosaic image (see $\S 6$ ).

We also searched the lower resolution $3.5^{\prime \prime}$ and $6^{\prime \prime}$ images for resolved sources not detected in the untapered $2^{\prime \prime}$ image above our completeness limit of $S_{p}=40 \mu \mathrm{Jy}$. This search yielded 57 additional sources in the tapered images above completeness limits of 50 $\mu \mathrm{Jy}$ at $3.5^{\prime \prime}$ resolution and $75 \mu \mathrm{Jy}$ at $6^{\prime \prime}$ resolution. Because the angular sizes are uncertain in these low signal to noise ratio detections (and in many cases may be instrumentally broadened), the adopted peak and integrated flux densities were set equal to the peak pixel values in the tapered images. These additional sources were added to the complete source list which in total contains 371 sources. The complete sample of all radio sources detected at $1.4 \mathrm{GHz}$ within $20^{\prime}$ of the phase center is presented in Table 2.

A description of Table 2 is as follows. All uncertainties are given at the one sigma level. Column (1) - The Right Ascension in J2000 coordinates with one sigma uncertainty.

Column (2) — The Declination in J2000 coordinates with one sigma uncertainty.

Column (3) — The deconvolved (FWHM) major axis of the best Gaussian fit to the source, $\Theta$, is given in arcsec.

Column (4) - The signal to noise ratio of the detection calculated from $S_{p} / \sigma$ where $S_{p}$ is the peak flux density as measured in either the $2^{\prime \prime}, 3.5^{\prime \prime}$, or $6^{\prime \prime}$ image, and $\sigma$ equals the rms noise in that image.

Column (5) - The integrated sky flux density $\left(\mathrm{S}_{1.4}\right)$ after correction for the instrumental gain, (see $\S 3.2)$,

with corresponding one sigma errors. 


\subsection{Instrumental Corrections}

We must also correct the derived source parameters for various instrumental effects. In order to measure the off axis response of the VLA to a point source, we observed the 4.2 Jy point source $1400+621$ in each of the cardinal directions at positions of 5, 10, 15, 20 and 25 arcmin from the nominal phase reference center.

There are four principal effects which reduce point source response as a function of radial distance from the phase center. In their degree of increasing importance they are 1) 3-D smearing, 2) finite time visibility sampling (time delay smearing), 3) chromatic aberration (bandwdith smearing), and 4) primary beam attenuation.

1. We have approximated the curvature of the celestial sphere with a number of two-dimensional facets. However, smearing may still be present at a reduced level near the edges of each of our individual facet images. The effect of a small amount of 3-D smearing are such that the integral flux density of a given source is preserved while its peak signal is reduced by an amount dependent on distance from the tangent point on the celestial sphere. In order to determine the amplitude of 3-D smearing in our data, we performed a series of simulations inserting point sources at a variety of distances from the phase tracking center into visibility data with our exact $(u, v)$ coverage. These data were then imaged in the same manner as the true sky images. In this manner we determined the amount of point source degradation as a function of distance from the celestial sphere tangent point or image facet center. At the maximum possible distance of a source from a tangent point $\left(\sim 600^{\prime \prime}\right)$ the peak degradation is less than $20 \%$. All values of the peak flux density $\left(S_{p}\right)$ were corrected for this effect according to our empirically fit polynomial.

2. The calibrated $(u, v)$ data set used to construct our images consisted of 13 sec averaged visibility data. Because the actual radio sources rotate in the sky during this sampling time, their flux density is smeared in the image plane. The analytic calculation 
of this smearing is complicated by the aspects of the observing geometry. However, at the North Celestial Pole the effect reduces to a tangential smearing in the image plane and its amplitude is given by $S_{p} / S_{t}=1-2.06 \times 10^{-9} \theta / \theta_{\text {syn }}$ where $S_{t}$ is the integrated flux density of a source located an angular distance $\theta$ from the phase tracking center and $\theta_{\text {syn }}$ is the size of the synthesized beam. We used this approximation to correct the peak flux densities as measured in our images.

3. Although we planned our observations of the HDF field to minimize the effects of chromatic aberration, for far-field sources this effect can still be important. It is especially crucial to understand the effects of smearing on the completeness level of the images. We measured the off-axis response of $1400+621$ due to chromatic aberration by examining the ratio of the peak to integrated flux density, $S_{p} / S_{i}$, as a function of distance from the image phase center. Bandwidth smearing is governed by the observing frequency, width and shape of the bandpass and the $(u, v)$ coverage, all of which define the synthesized beam. In theory, these are known functions and the final beam response can be calculated analytically as a function of position in the image. However, in practice such uncertainties as non-uniform $(u, v)$ coverage, central intermediate frequency offsets, and imperfect bandpass filters make this impossible. We chose instead to fit a function to the empirical data of the form $S_{p} / S_{i}$ $=\left(1+(\mathrm{r} / \mathrm{k})^{2}\right)^{-0.5}$ where $\mathrm{r}$ is the distance from the phase center and $\mathrm{k}$ is a constant which absorbs the uncertainties discussed above. Our least squares fit to the empirical data is shown in Figure 3 where $\mathrm{k}=16.19$ arcmin. This is within $3 \%$ of the theoretical value assuming $\delta \nu / \nu=3.125 / 1400.5$ and a $\theta_{\text {beam }}=2.1^{\prime \prime}$. For purposes of correcting $S_{p}$ in our images, we use the above equation scaled by the appropriate beam size. The integrated flux density, $S_{i}$ is preserved and needs no correction for smearing.

4. The primary beam attenuation at $1.40 \mathrm{GHz}$ has been measured to about $1 \%$ (Condon 1997) to the first sidelobe $(-10 \mathrm{~dB})$. Each of the peak and integrated flux densities 
in the source list were corrected for the primary beam attenuation. The uncertainty in the correction is the standard rms pointing error of the VLA elements (about $15^{\prime \prime}$ ) multiplied by the differential log of the primary beam response.

In Figure 3 we plot the point source response of a source as a function of distance from the phase center. We define the instrumental gain factor as the product of bandwidth smearing, time averaging smearing, and the primary beam correction.

\subsection{Positional Accuracy}

The assumed position of our phase calibrator $1400+621$ is $\alpha=14^{h} 00^{m} 28.6526^{s}$ and $\delta=62^{\circ} 15^{\prime} 38.526^{\prime \prime}(\mathrm{J} 2000)$. We observed systematic, monotonically increasing shifts in both RA and DEC of comparable amplitude as a function of $r$. It is a small effect and the difference between the actual radial distance from the field center and that measured in the image plane is $\delta \mathrm{r}=-0.042$ arcsec at 25 arcmin distance from the field center.

This small systematic term can be explained by the so called annual aberration effect (Fomalont et al. 1992). The predicted scale contraction from annular aberration in the direction of the HDF during the Julian epoch 1996.6 is 0.9999783. The good agreement between the observed and predicted scaling factor for $1400+621$ yields confidence that our images are free from significant distortions due to asymmetric bandpasses or IF offsets.

We also corrected all the radio source positions in our catalog for position offsets induced by the $3-\mathrm{D}$ effect as discussed in $§ 3.2$. By phase shifting the image tangent points to the location of individual sources, their true angular positions on the sky were measured. The difference between the apparent position as measured on the nominal sky maps and the corrected positions is typically only $0.2^{\prime \prime}$ in the north-south direction.

After correction for annular aberration and the 3-D term, the relative positional 
accuracy for sources across the field in the limit of infinite signal to noise should approach about $0.02^{\prime \prime}$. The absolute positional accuracy depends on the translation of our phase calibrator position to that of the HDF and is about $0.02^{\prime \prime}$. Thus we estimate our radio catalog to be within $0.03^{\prime \prime}$ of the J2000/FK5 coordinate grid. The single coordinate rms position errors as given in Table 2 are defined as $\sigma=\sqrt{\left(1.8^{\prime \prime} / 2 \sigma_{\text {snr }}\right)^{2}+\left(0.03^{\prime \prime}\right)^{2}}$.

\section{Survey Completeness and Source Counts}

Because the completeness of the radio source sample is defined in terms of peak image

flux, $S_{p}$, corrections must be made for the the instrumental response and biases inherent in our detection algorithm. Although we have corrected the individual sources in Table 2 for these effects, we now calculate the fraction of sky sources which remain undetected in our survey due to the finite angular size of the sources.

\subsection{Angular Size Distribution}

Previous high resolution studies of the microjansky radio population suggested that the median angular size $\left(\theta_{\text {med }}\right)$ for submillijansky radio sources is approximately $2^{\prime \prime}$ and almost independent of flux density between 80 - $1000 \mu \mathrm{Jy}$ (Windhorst et al. 1993, Fomalont et al. 1991, Oort 1988). The resolution of our present microjansky survey is thus well suited to study the angular size distribution for a statistically large and well defined sample of microjansky radio sources.

Of the 151 radio sources in our complete sample with $70 \mu \mathrm{Jy}<S_{i}<1000 \mu \mathrm{Jy}$ for which we have angular size information, only 77 (50\%) are resolved with our typical $2^{\prime \prime}$ resolution limit. We divided these sources into two flux density bins, containing approximately equal numbers of resolved sources. Considering only the number of radio sources with angular 
size greater than $2.7^{\prime \prime}$ (the angular size detection limit of the weakest radio sources in our sample as defined in $§ 3.1)$, we find twice as many resolved sources with $S_{i}>250 \mu \mathrm{Jy}(52 \%)$ as opposed to those with $S_{i} \leq 250 \mu \mathrm{Jy}(25 \%)$. This suggests that $\theta_{\text {med }}$ may be a decreasing function of flux density. Figure 4 shows our measurements of angular size as a function of source intensity. In order to estimate the mean angular size of this sample, we applied the survival analysis techniques of Feigelson \& Nelson (1985) using the statistical package ASURV (Rev. 1.2; Isobe \& Feigelson 1992). This technique incorporates upper limits in the calculation of the mean of a distribution, which is particularly important in our sample which is dominated by non-detections (i.e. we are measuring the tail of the angular size distribution). The technique assumes a symmetric Gaussian model, hence the mean and median are equal. At $S_{1.4}=370 \mu \mathrm{Jy}, \theta_{\text {med }}=2.6^{\prime \prime} \pm 0.4^{\prime \prime}$, and at $S_{i}=100 \mu \mathrm{Jy}, \theta_{\text {med }}=$ $1.6^{\prime \prime} \pm 0.3^{\prime \prime}$. The errors are based on the number of angular size measurements (not limits).

In Fig. 5 , we compare our determinations of $\theta_{\text {med }}$ with previous measurements made at $1.4 \mathrm{GHz}$. Because of the uncertain selection effects inherent in the higher frequency deep radio surveys, particularly their bias towards flat-spectrum, compact AGN, we chose not to include these points. With the notable exception of the discrepant Condon \& Coleman (1985) point, there is general agreement amongst the different data. Because the median angular size is known to change rather sharply below a few millijansky at $1.4 \mathrm{GHz}$ (presumably due to the emergence of an increasing population of starburst galaxies among radio sources) from $\sim 10^{\prime \prime}$ to a few arcsec, we suggest that the high flux density point of Oort (1988) is too low, possibly due to resolution biases in his A-array snapshots.

We believe the decrease in angular size at lower flux densities to be real. Thus for the purposes of modeling the median angular size-flux density relationship, we fit a function of the form:

$$
\theta=\operatorname{frac} 34 \times 0.175 S_{1.4}^{0.5}+f r a c 14 \times 0.1 \operatorname{arcsec}
$$


This fit is also shown in Fig. 5. For completeness, we also plot a straight line, with $\theta$ $=2.0^{\prime \prime}$ and independent of flux density.

\subsection{Completeness}

In order to investigate the combined effects of noise, population, and resolution bias on the completeness level of our survey, we used a series of Monte Carlo simulations similar to the ones described in $§ 3.1$. In particular we want to determine how many sources with $S_{i} \geq$ $40 \mu \mathrm{Jy}$, but with $S_{p}<40 \mu \mathrm{Jy}$ we missed based on our peak flux density detection limit. We randomly populated our sky images with 100 sources of finite angular size assuming an angular size distribution as found in $§ 4.1$. This simulation was repeated at a variety of flux densities from 40 to $1000 \mu \mathrm{Jy}$. In each flux density interval the ratio of the number of sources recovered from the images with $S_{p} \geq 40 \mu \mathrm{Jy}$ to the number originally injected in the model was tabulated. This was taken to be the effective correction factor needed to account for the combined effects of resolution, population and noise bias in our images (although resolution bias is always the dominant term). At $80 \mu \mathrm{Jy}$ which is the average flux density source detected in our survey (weighted by $S^{-2.4}$ ) and where the count will most accurately be determined, this correction factor is 1.05. As resolution bias is the dominant source of incompleteness in our survey, we estimate that we have detected approximately 95\% of the microjansky sources in the HDF region to this flux density limit. The principal uncertainty in the correction factor is the uncertainity in the angular size distribution of the microjansky radio sources. If we had assumed a constant $\theta=2.0^{\prime \prime}$ model, our corrections would have increased by over a factor of two.

From the complete source list of Table 2, we then binned sources in flux density intervals such that each bin had at least 50 radio sources (except for the highest flux density bin). The differential count was then calculated based on the number of sources in each bin 
interval. Bandwidth smearing, time averaging smearing, and the primary beam response decreases the effective area over which a source of given $S_{p}$ can be detected. Thus when counting the number of sources in each bin, care must be taken to weight the contribution of each source to the count by the effective area over which it could have been detected. This factor can be calculated by solving for the image radius where a source of amplitude $S_{p}$ would have just been missed by our peak detection limit (Katgert 1973). Table 3 presents the differential source counts for our complete flux limited sample. The counts from this survey are compared to other microjansky surveys in Figure 6a and 6b (Mitchell \& Condon 1985, Oort \& Windhorst 1985, Hopkins et al. 1998) normalized to a Euclidean geometry

$\mathrm{n} / n_{o}=n(s) / S^{-2.5}$. In general the agreement is reasonable and in agreement with the errors and possible field to field variations. The best fit to the source counts in this field in the range $40-1000 \mu \mathrm{Jy}$ is $n(S)=(8.25 \pm 0.42) S^{-2.38 \pm 0.13} \mathrm{ster}^{-1} \mathrm{Jy}^{-1}$.

The counts in the HDF appear systematically lower than those of other fields above $100 \mu \mathrm{Jy}$. This effect could be due either to 1) real field to field variations on the degree scale as a result of large-scale clustering of radio sources, or 2) survey incompleteness due to the finite angular size of the radio sources. Without complementary, low resolution observations, it is difficult to discriminate between these two possibilities. We note that if the mean angular size does not decrease significantly below $100 \mu \mathrm{Jy}$, the radio sky will become forever naturally confused at the level of a few hundred nanojansky, perhaps providing a natural limitation to the sensitivity of the next generation of centimeter radio telescopes (Windhorst et al. 1993).

\section{Spatial Clustering}

In order to test for the presence of two dimensional spatial clustering among the radio sources in the Hubble Deep Field, we calculated the two-point correlation function for the 
sources in Table 2. First, we compiled a table of angular separations by considering the separation of each individual source with all other sources in the catalog. These provide our $D D$ estimate (Peebles, 1980). Next, we generated random catalogs of sources according to the source count of $\S 4.2$, and distributed randomly across a $40^{\prime}$ VLA primary beam. The peak flux densities of these sources have been attenuated by the instrumental corrections discussed in §3.2. Angular pairs were calculated for these catalogs and form the basis of our $R R$ measurement. We define the correlation function of our catalog to be $w(\theta)=\mathrm{DD} / \mathrm{RR}$ -1 . The correlation function of our catalog on scales of $0^{\prime}-40^{\prime}$ is presented in Table 4 .

We calculated the errors in our clustering measurement by following the bootstrap method of Ling et al. (1986). These agree well with the Poissonian error estimate of $\delta w(\theta)$ $=1+w(\theta)) / \mathrm{N}_{D D}$ where $\mathrm{N}_{D D}$ is the number of independent data pairs in a given bin. We find evidence for an excess of radio sources on scales of approximately $1-10^{\prime}$, while on scales much larger than this there are fewer radio sources in our catalog than expected from a random distribution. Figure 7 shows the correlation function for radio sources in the HDF, compared to the correlation function of a somewhat shallower $1.4 \mathrm{GHz}$ survey of Oort (1987) complete to $100 \mu \mathrm{Jy}$. The amplitudes are comparable in the two separate surveys.

Spatial clustering at higher flux density levels and lower amplitudes has been reported by Cress et al. (1996) and Magliocchetti et al. (1998). More recently, Hopkins et al. (1999) claim fluctuations in field to field source counts at similar completeness levels to ours, possibly indicating the presence of large-scale radio source spatial variations. Thus it is plausible that there are both fewer radio sources in the HDF region than the average field, and that these sources are clustered on arcmin scales amongst themselves. 


\section{Radio Spectral Indices}

The HDF has been observed previously with the VLA at $8.5 \mathrm{GHz}$ to a one sigma sensitivity of $1.8 \mu \mathrm{Jy}$ (Paper I). In June 1997 we observed the HDF region for an additional 40 hours at $8.5 \mathrm{GHz}$. We mosaiced an area defined by four separate pointings offset $2.7^{\prime}$ from the center of the HDF (the half-power scale of the primary beam response) in each of the cardinal directions for about 10 hours duration each. The observing technique and data reduction are discussed in Paper I. The final combined $8.5 \mathrm{GHz}$ images have an effective resolution of $3.5^{\prime \prime}$ and a completeness limit of $8 \mu \mathrm{Jy}$. The sensitivity of this mosaic to sky emission is a sharp function of distance from the nominal pointing center because the observations were heavily weighted towards imaging the central HDF region.

Because the size of the VLA primary beam scales inversely with frequency, our sensitivity at $8.5 \mathrm{GHz}$ is limited to the inner 6.6 arcmin (HWHM) of the $1.4 \mathrm{GHz}$ field. This is the point where the maximum beam attenuation at $8.5 \mathrm{GHz}$ is equal to 0.2 (while at $1.4 \mathrm{GHz}$ the attenuation is only 0.9 ). Within this region there are 109 sources contained in the $1.4 \mathrm{GHz}$ complete sample. We measured the $8.5 \mathrm{GHz}$ flux density at the location of each of these sources. When a source was not clearly detected $\left(S_{p}<3 \sigma\right.$ at $\left.8.5 \mathrm{GHz}\right)$, we calculated a conservative upper limit to its $8.5 \mathrm{GHz}$ flux density equal to three times the rms noise corrected by the antenna gain. If a $1.4 \mathrm{GHz}$ radio source had a peak flux value $3 \sigma<S_{p}<5 \sigma$, its flux limit was taken as $S_{p}$ also corrected by the primary beam. This ensures that our 1.4 GHz selected spectral index sample is complete and free from uncertain weak source biases. Based on this criteria, 30 sources from the $1.4 \mathrm{GHz}$ sample had clear counterparts in the $8.5 \mathrm{GHz}$ image. Using the $1.4 \mathrm{GHz}$ and $8.5 \mathrm{GHz}$ flux density values as measured in their respective $3.5^{\prime \prime}$ convolved images, we calculated individual spectral indices using the convention $S_{\nu} \propto \nu^{-\alpha}$. In the following discussion, steep spectrum sources are defined as those with $\alpha \geq 0.50$, while flat spectrum as those with $\alpha<0.50$. 
The $1.4 \mathrm{GHz}$ to $8.5 \mathrm{GHz}$ spectral index distribution of the $1.4 \mathrm{GHz}$ selected sample is shown in Figure 8 (only those with meaningful lower limits, $\alpha \geq 0.50$ are shown for clarity). Because of the large number of spectral index limits for the $1.4 \mathrm{GHz}$ sample $(79 / 109)$, we chose to only consider those sources with $S_{1.4}>100 \mu \mathrm{Jy}$ when calculating the mean of the sample. The mean as calculated from both the detections and lower limits (using ASURV) for sources with $S_{1.4}>100 \mu \mathrm{Jy}$ is $\bar{\alpha}_{1.4}=0.85 \pm 0.16$. For those sources with $S_{1.4}>100$ $\mu \mathrm{Jy}$ the fraction of steep spectrum sources is $\alpha=0.62$. We also calculated the median of the spectral index distribution for the entire 1.4 GHz sample. We did not consider spectral index lower limits which were weaker than $\alpha_{1.4}>0.3$, to avoid a bias in the median calculation. The median of the $1.4 \mathrm{GHz}$ selected sample is 0.63 .

We now consider those radio sources within the central $6.6^{\prime}$ (HWHM) detected on the basis of the $8.5 \mathrm{GHz}$ data alone. There are 29 sources in the complete sample $8.5 \mathrm{GHz}$ sample of Paper I. Eleven additional sources were detected in the mosaiced regions with $S_{p} \geq 5 \sigma$. These 40 sources comprise a complete sample of radio sources detected at $8.5 \mathrm{GHz}$ within $6.6^{\prime}$ of the HDF center. We measured the $1.4 \mathrm{GHz}$ flux densities at their positions in the $3.5^{\prime \prime}$ image. All but 10 of these sources are contained in the complete $1.4 \mathrm{GHz}$ sample in Table 2. Upper limits to the $1.4 \mathrm{GHz}$ flux density were calculated as three times the rms normalized by the antenna gain.

The spectral index distribution is shown in Figure 9. The mean spectral index for the $8.5 \mathrm{GHz}$ selected sample is $\bar{\alpha}_{8.5}=0.35 \pm 0.07$ while the median spectral index of the detections is 0.41 . The fraction of flat spectrum sources in the $8.5 \mathrm{GHz}$ selected sample is 0.60 . Table 5 gives individual spectral indices or limits (where meaningful limits are available) for both the 1.4 and $8.5 \mathrm{GHz}$ samples. 


\subsection{The Nature of Flat Spectrum Sources: AGN vs. Starbursts}

It has been noted by previous authors that below a few milljansky, the median spectral index for high frequency selected samples $(\nu \geq 5 \mathrm{GHz})$ flattens from a value of 0.7 to about 0.3-0.4 and then remains constant for at least two decades in flux density (Windhorst et al. 1993 and references therein). Our spectral index study confirms this trend.

This raises the question of what physical mechanism is responsible for the flattening of the high frequency selected microjansky population. One clue comes from the optical identification of the sources. Of the 26 flat spectrum sources presented here, only 4 can be reliably associated with elliptical galaxies, the majority (70\%) residing in mergers, interacting disk systems, or isolated field spirals (Paper I, Richards et al. 1998). Thus the flattening of the spectral index distribution for the microjansky population is unlikely to be due to radio evolution of the elliptical population.

If the $8.5 \mathrm{GHz}$ sample preferentially selects out self-absorbed AGN cores from the microjansky population, then we might expect these sources to have a smaller angular size on average as compared to a $1.4 \mathrm{GHz}$ selected sample. The mean angular size for the 26 flat $(\alpha<0.5)$ spectrum sources of Table 5 , is $\theta=1.7 \pm 0.6$, as compared to $\theta=1.8 \pm 0.5$, for the 47 steep $(\alpha \geq 0.5)$ spectrum sources. Thus there is no evidence for a significant change in source size between the flat and steep spectrum population. Interestingly, there are only four inverted spectrum sources among the flat spectrum sample, indicating that strongly self-absorbed systems are rare among the microjansky population.

In Paper I we considered two possibilities for the origin of microjansky radio emission in distant disk galaxies, 1) increased radio activity associated with a central engine (e.g., Seyfert and LINER AGN), and 2) radio emission excited by star-formation. Both are capable of producing flat radio spectra through synchrotron self-absorption in the case of the former, and through increasing amounts of thermal radio emission in the later. These 
two different physical mechanisms take place on very different physical scales. For the case of star formation these scales correspond from approximately 0.1-10 kpc as observed in the local starburst population (Condon 1989). Thus the cosmological microjansky population at a mean redshift of 0.8 (Paper I) should have an angular extent of $0.01-1^{\prime \prime}$ if star formation is the ultimate source of energy powering the radio emission. On the other hand if the radio emission has its origin in an AGN then the flattening of the spectral index distribution can be attributed to partial self-absorption. In this case the observed flux density of the source makes calculation of a minimum angular size for synchrotron self-absorption possible (Pacholczyk 1970). For a $100 \mu \mathrm{Jy}$ source with a critical absorption frequency of $1.4 \mathrm{GHz}$ and an assumed magnetic field strength no larger than $10^{-4}$ Gauss, the characteristic angular size scale is of order $10^{-5}$ arcsec.

Because the radio spectral index is such a sensitive function of absorption varying from -2.5 in the case of pure synchrotron self-absorption, to 0.8 for a standard transparent, non thermal spectrum, the observation of low dispersion in the flat spectral indices of the microjansky population (i.e., very few inverted spectrum sources) would suggest that we are seeing very nearly the same fraction of absorbed radiation in all sources. Perhaps a more natural explanation for the observed spectral index distribution of flat spectrum microjansky radio sources is that we are observing varying ratios of thermal to synchrotron emission causing the spectral indices to vary from -0.1 to 0.8 (cf., Condon 1992). This could be due to the combined effect of a radio K-correction which serves to bring a greater fraction of bremsstrahlung radiation into the observed radio window for sources at appreciable redshifts, as well as a steepening of the synchrotron radiation itself as a result of synchrotron and Compton losses off the microwave background. A flatenning of the relativistic electron energy spectrum could flatten the observed synchrotron spectral index as well. Sub-arcsecond radio observations of the microjansky population are necessary to discriminate between these possibilities (Muxlow et al. 1998). High frequency observations 
would also be useful to determine the slope of the radio spectrum in these distant radio sources, but will not be feasible until the commisioning of the Millimeter Array or until the expansion of the high frequency capabilities of the VLA.

\section{Conclusions}

We have presented a complete catalog of 371 radio sources brighter than $40 \mu \mathrm{Jy}$ at 1.4 GHz in a $0.3 \mathrm{deg}^{2}$ field centered on the Hubble Deep Field. This is the most sensitive survey available at this resolution $\left(2^{\prime \prime}\right)$ and frequency. For a subsample of these sources we have calculated two point spectral indices based on $8.5 \mathrm{GHz}$ mosaic observations.

The principal results of this study are:

1. We have extended the direct source count at $1.4 \mathrm{GHz}$ to $40 \mu \mathrm{Jy}$, confirming that the differential slope for radio sources remains steep at $\gamma=-2.4$ to this level.

2. The average angular size for the microjansky population is observed to decrease as a function of flux density. The mean size for radio sources between $40-1000 \mu \mathrm{Jy}$ is $2.0^{\prime \prime}$, consistent with their association with large disk galaxies at $z \sim 1$.

3. Microjansky radio sources appear to be clustered on scales of $1^{\prime}-40^{\prime}$, corresponding to projected distances of $0.5-20 \mathrm{Mpc}$.

4. The average spectral index for a $1.4 \mathrm{GHz}$ selected subsample is $\bar{\alpha}_{1.4}=0.85 \pm 0.2$, indicating optically thin synchrotron emission as the dominant radio emission mechanism. For a $8.5 \mathrm{GHz}$ selected sample, the mean is $\bar{\alpha}_{8.5}=0.4 \pm 0.1$. This flattening of the spectral index distribution over that of samples selected above a few millijansky is consistent with either the cosmological evolution of the disk galaxy AGN population (LINERs and Seyferts) or of their star-formation activity. We suggest that we are observing increasing amounts 
of bremsstrahlung radiation in these sources, causing the observed decrease in the spectral index distribution at $8.5 \mathrm{GHz}$.

I thank my collaborators Ed Fomalont, Ken Kellermann, Bruce Partridge, Rogier Windhorst, and Tom Muxlow for their help with this project. This study would not have been possible without the expert assistance of the NRAO staff in the planning, execution, and analysis of these observations, especially F. Owen and M. Rupen. This work benefited from suggestions by J. Condon and J. Wall. I also thank L. Cowie and A. Barger for pointing out a positional inconsistency in an earlier version of this work. 


\section{References}

Cillegi, P. et al. 1998, MNRAS, in press

Clark, B. G. 1980, A\& A, 89, 377

Coleman,P. H. \& Condon, J. J. 1985, AJ, 90, 1431

Condon, J. J. 1989, ApJ, 338, 13

Condon, J. J. 1992, ARA \& A, 30, 575

Condon, J. J. 1997, PASP, 109, 166

Cornwell, T. J. \& Perley, R. A. 1992, A \& A, 261, 353

Cress, C., Helfand, D., Becker, R., Gregg, M. \& White, R. 1996, ApJ, 473, 7

Donnelly, R. H., Partridge, R. B \& Windhorst, R. A. 1987, ApJ, 321, 94

Feigelson, E. D. \& Nelson, P. I. 1985, ApJ, 293, 192

Fomalont, E. B., Windhorst, R. A., Kristian, J. A. \& Kellermann, K. I. 1991, AJ, 102, 1258

Fomalont, E. B., Goss, W. M., Lyne, A. G., Manchester, R. N. \& Justtanont, K. 1992, MNRAS, 258, 497

Fomalont, E. B., Kellermann, K. I., Richards, E. A., Windhorst, R. A. \& Partridge, R. B. 1997, ApJL, 475, 5

Gruppioni, C., Zamorani, G., De Ruitter, H. R., Parma, P, Mignoli, M. \& Hasinger, G 1997, MNRAS, 286, 470

Hammer, F., Crampton, D., Lilly, S. J., LeFevre, O. \& Kenet, T. 1995, MNRAS, 276, 1085

Hopkins, A., Afonso, J., Cram, L. \& Mobasher, B. 1999, ApJL, in press 
Hopkins, A. M., Mobasher, B., Cram, L. \& Rowan-Robinson, M. 1998, MNRAS, 296, 839

Isobe, T. \& Feigelson, E. D. 1991, ASURV, Rev. 1.2

Katgert, P., Oort, J. \& Windhorst, R. 1988, A\& A, 195, 21

Ling, E. N., Frenk, C. S. \& Barrow, J. D. 1986, MNRAS, 223, 21p

Magliocchetti, M., Maddox, S., Lahav, O. \& Wall, J. 1998, MNRAS, 300, 257

Mitchell, K. J. \& Condon, J. J. 1985, AJ, 90, 1987

Muxlow et al. 1999, in preparation

Oort, J. A. 1987, A \& AS, 71, 2210

Oort, J. A. 1988, A \& A, 193, 50

Oort, J. A. \& Windhorst, R. A. 1985, A\&A, 145, 4050

Pacholczyk 1970, Radio Astrophysics (San Francisco: W. H. Freeman)

Peebles, P. J. E. 1980, The Large Scale Structure of the Universe (Princeton: Princeton University Press)

Richards, E. A. 1996, in IAU 175: Extragalactic Radio Sources, eds., Ekers, R., Fanti, C. \& Padrielli, L., 593

Richards, E. A., Kellermann, K. I., Fomalont, E. B., Windhorst, R. A., \& Partridge, R. B. 1998a, AJ, 116, 1039 (Paper I)

Richards, E. A. 1999b, et al., in preparation (Paper III)

Rowan-Robinson, M. et al. 1997, MNRAS, 289, 490

Windhorst, R. A, Fomalont, E. B., Kellermann, K. I., Partridge, R. B., Richards, E. A., Franklin, B. E., Pascarelle, S. M. \& Griffiths, R. E. 1995, Nature, 375, 471 
Windhorst, R. A., Fomalont, E. B., Partridge, R. B. \& Lowenthal, J. D. 1993, ApJ, 405, 498

Windhorst, R. A., van Heerde, G. M. \& Katgert, P. 1984, A \& AS, 58, 1 


\section{Figure Captions}

1. A greyscale of the inner $9.5^{\prime} \times 9.5^{\prime}$ of the $1.4 \mathrm{GHz}$ image. The pixel range is from $-10-20 \mu \mathrm{Jy}$. The effective resolution is $1.8^{\prime \prime}$ with an rms noise of $7.5 \mu \mathrm{Jy}$. The image has been corrected by the primary beam attenuation.

2. Here we show the same $9.5^{\prime} \times 9.5^{\prime}$ area at $8.5 \mathrm{GHz}$. The greyscale extends from $-2-$ $6 \mu \mathrm{Jy}$. The effective resolution is $3.5^{\prime \prime}$ with an $\mathrm{rms}$ noise of $1.6 \mu \mathrm{Jy}$. This image has been corrected by the primary beam attenuation and hence the noise is not uniform.

3. The total off-axis response of a point source due to the combined effects of bandwidth smearing, time averaging smearing, and the primary beam correction (gain). The relative

amplitude decrease due to time average smearing (tsmear), bandwidth smearing (bwsmear), and the primary beam attenuation (pbcor) are also shown.

4. The angular size distribution for the $1.4 \mathrm{GHz}$ complete sample. Notice the increasing number of upper limits at lower flux density levels.

5. The $1.4 \mathrm{GHz}$ median angular size vs. flux density relation for microjansky radio sources. Points from Oort (1988; O88), Coleman \& Condon (1985; CC), and this study are plotted. The curve shown has been calculated according to the model given in $\S 4$. The broken line represents a median angular size independent of flux density.

6a. The $1.4 \mathrm{GHz}$ source counts from this field and other deep radio surveys are shown. The count is presented in differential form normalized to the counts expected in a Euclidean geometry. For comparison, counts from Mitchell \& Condon (1985; MC), the Phoenix Deep Field (Hopkins et al. 1998; PDF), Oort \& Windhorst (1985, OW85), and the ELAIS survey (Cillegi et al. 1998; ELAIS) are plotted. The best fit to the earlier deep survey compilation by Katgert et al. (1988) is shown as a solid line.

6b. A blow-up of the sub-millijansky counts. The solid line is the best fit to the 
current survey data. There is good evidence for field to field fluctuations above that of the statistical noise.

7. The correlation function of the HDF radio sruvey is shown as heavy dots. The correlation function in the Lynx3 survey of Oort (1987) is shown for comparison.

8. The spectral index flux density distribution for the $1.4 \mathrm{GHz}$ selected sample. The mean of the sample as calculated by survival analysis is shown as the broken line.

9. The spectral index flux density distribution for the $8.5 \mathrm{GHz}$ selected sample. The mean of the sample as calculated by survival analysis is shown as the broken line. 


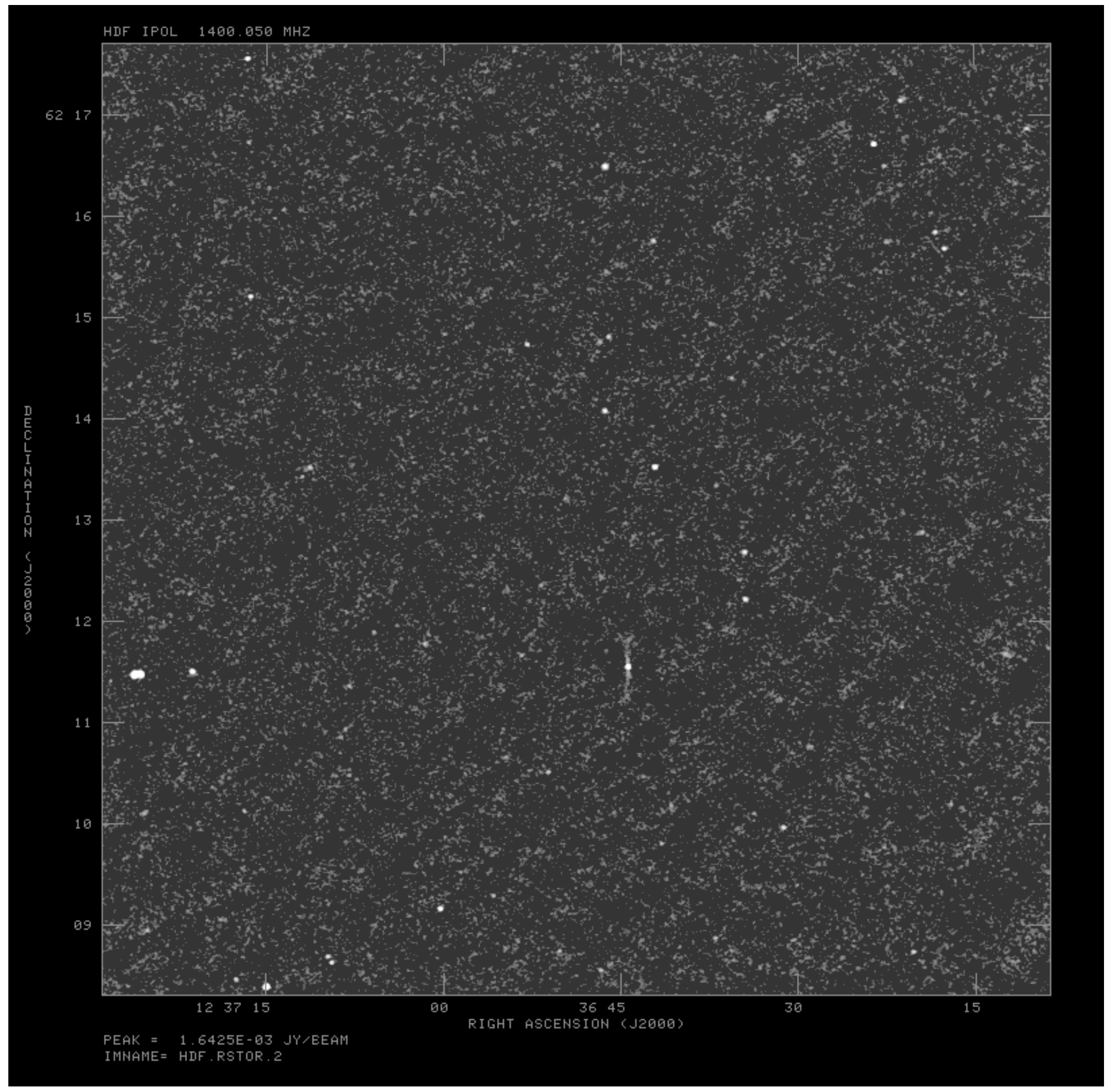




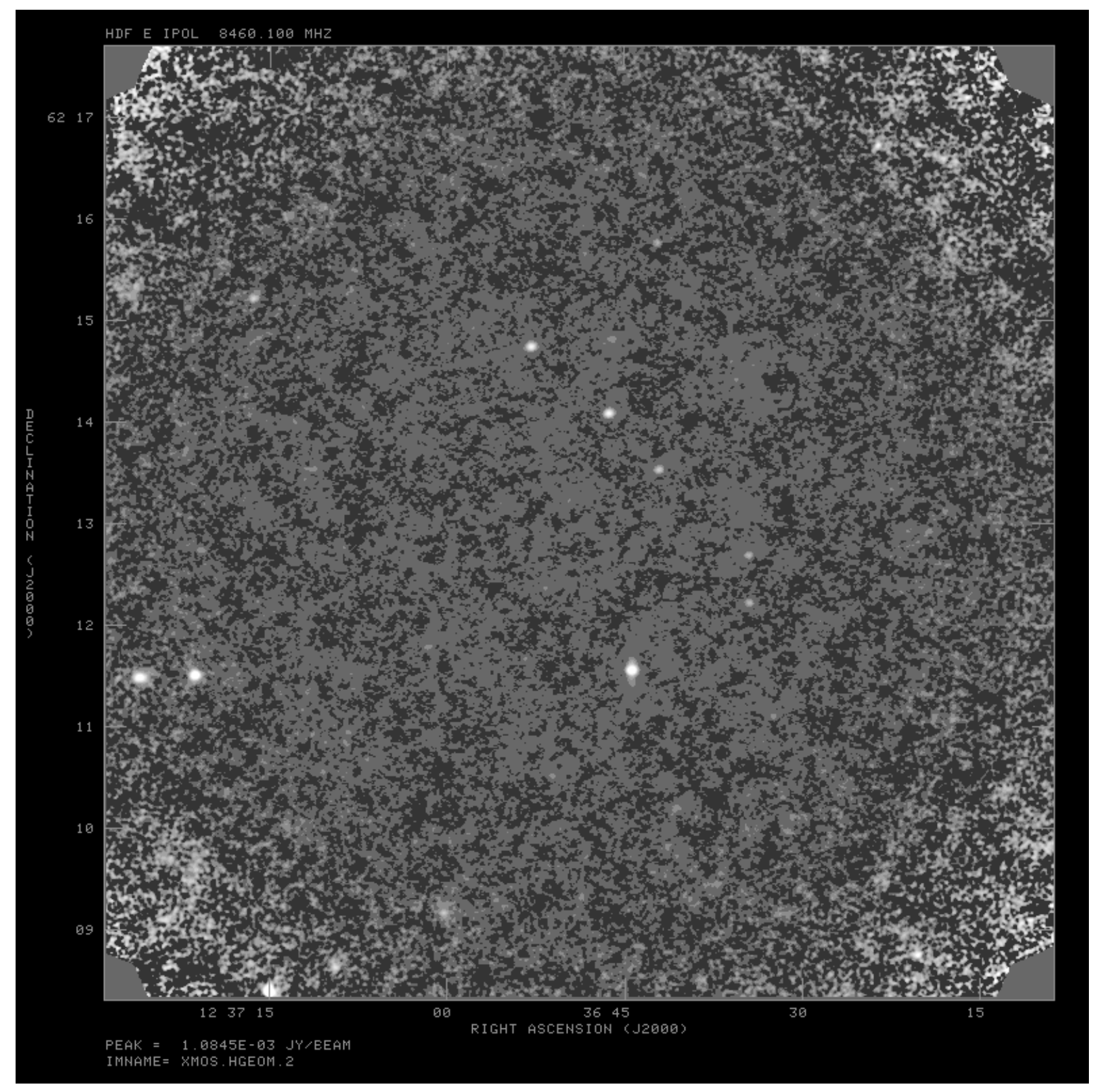



Table 2: Complete $1.4 \mathrm{GHz}$ Source List

\begin{tabular}{|c|c|c|c|c|c|c|c|}
\hline $\begin{array}{l}\text { Right Ascension } \\
h h m m \text { ss }\end{array}$ & & $\begin{array}{c}\text { Declination } \\
0, \prime\end{array}$ & & $\left(^{\theta}\right.$ & $\sigma_{s n r}$ & $\begin{array}{r}S_{1.4} \\
(\mu \mathrm{Jy})\end{array}$ & \\
\hline 123336.583 & 0.015 & 623019.72 & 0.10 & 6.5 & 48.7 & 50900.0 & 2560.0 \\
\hline 123400.107 & 0.027 & 621222.16 & 0.19 & - & 5.7 & 260.0 & 28.3 \\
\hline 123403.519 & 0.017 & 621420.75 & 0.12 & $<2.1$ & 15.5 & 693.0 & 42.2 \\
\hline 123410.657 & 0.019 & 620615.96 & 0.13 & 3.1 & 10.7 & 629.0 & 40.2 \\
\hline 123411.242 & 0.015 & 621616.78 & 0.11 & 11.7 & 27.3 & 9700.0 & 487.0 \\
\hline 123414.339 & 0.028 & 622109.18 & 0.19 & - & 5.4 & 253.0 & 36.2 \\
\hline 123416.118 & 0.021 & 620722.75 & 0.15 & 3.1 & 8.1 & 453.0 & 31.7 \\
\hline 123423.970 & 0.022 & 622038.23 & 0.16 & 3.9 & 7.5 & 394.0 & 29.2 \\
\hline 123426.705 & 0.015 & 621331.90 & 0.11 & 3.2 & 24.9 & 1350.0 & 69.9 \\
\hline 123426.855 & 0.015 & 621454.63 & 0.10 & $<2.0$ & 45.1 & 2710.0 & 140.0 \\
\hline 123428.656 & 0.027 & 621827.80 & 0.19 & - & 5.6 & 180.0 & 26.3 \\
\hline 123429.878 & 0.015 & 621806.62 & 0.10 & 3.0 & 29.6 & 1350.0 & 69.8 \\
\hline 123431.903 & 0.024 & 621950.98 & 0.16 & - & 6.9 & 235.0 & 22.1 \\
\hline 123434.706 & 0.023 & 622022.98 & 0.16 & - & 7.2 & 224.0 & 21.7 \\
\hline 123435.807 & 0.023 & 622439.63 & 0.16 & - & 7.3 & 320.0 & 35.9 \\
\hline 123451.106 & 0.022 & 620758.16 & 0.15 & - & 7.6 & 165.0 & 16.4 \\
\hline 123451.464 & 0.021 & 620548.28 & 0.15 & - & 8.6 & 204.0 & 18.4 \\
\hline 123451.724 & 0.027 & 615850.06 & 0.19 & - & 5.6 & 269.0 & 28.2 \\
\hline 123452.278 & 0.014 & 620235.70 & 0.10 & 15.2 & 2500 & 293000.0 & 14600.0 \\
\hline 123456.990 & 0.023 & 622527.00 & 0.16 & - & 7.3 & 172.0 & 22.0 \\
\hline 123500.128 & 0.018 & 621101.00 & 0.13 & $<2.1$ & 12.0 & 211.0 & 16.1 \\
\hline 123505.666 & 0.023 & 621632.28 & 0.16 & - & 6.9 & 115.0 & 13.2 \\
\hline 123506.051 & 0.018 & 622756.25 & 0.13 & 3.3 & 11.6 & 730.0 & 43.3 \\
\hline 123506.555 & 0.026 & 620707.92 & 0.18 & - & 6.0 & 106.0 & 13.7 \\
\hline 123506.785 & 0.023 & 621639.55 & 0.16 & - & 7.1 & 116.0 & 13.2 \\
\hline 123509.078 & 0.025 & 621055.29 & 0.17 & - & 6.3 & 96.6 & 12.3 \\
\hline 123509.137 & 0.024 & 621733.60 & 0.17 & - & 6.8 & 94.6 & 12.8 \\
\hline 123509.611 & 0.017 & 620855.80 & 0.12 & 3.0 & 12.9 & 307.0 & 19.3 \\
\hline 123509.918 & 0.026 & 622056.13 & 0.18 & - & 6.1 & 117.0 & 14.6 \\
\hline 123510.276 & 0.015 & 622201.80 & 0.10 & $<2.0$ & 42.8 & 1880.0 & 98.6 \\
\hline 123510.651 & 0.015 & 621937.24 & 0.11 & $<2.0$ & 28.1 & 493.0 & 27.8 \\
\hline 123513.145 & 0.024 & 620802.82 & 0.17 & - & 6.7 & 105.0 & 12.7 \\
\hline 123513.589 & 0.022 & 621240.10 & 0.16 & - & 7.5 & 111.0 & 12.1 \\
\hline 123513.729 & 0.017 & 620918.63 & 0.12 & $<2.1$ & 14.5 & 215.0 & 15.5 \\
\hline 123514.590 & 0.023 & 621736.62 & 0.16 & - & 7.0 & 106.0 & 12.5 \\
\hline 123514.937 & 0.016 & 621509.85 & 0.11 & $<2.0$ & 18.7 & 266.0 & 17.2 \\
\hline
\end{tabular}




\begin{tabular}{|c|c|c|c|c|c|c|c|}
\hline 123515.165 & 0.020 & 621013.41 & 0.14 & - & 9.0 & 128.0 & 12.6 \\
\hline 123516.197 & 0.015 & 621636.30 & 0.10 & $<2.0$ & 39.2 & 563.0 & 30.3 \\
\hline 123518.667 & 0.023 & 621854.94 & 0.16 & - & 7.4 & 113.0 & 12.8 \\
\hline 123519.398 & 0.021 & 622024.98 & 0.15 & - & 8.2 & 137.0 & 13.9 \\
\hline 23521.190 & 0.021 & 615926.19 & 0.14 & 3.4 & 8.6 & 378.0 & 25.9 \\
\hline 123521.622 & 0.014 & 621807.07 & 0.10 & $<1.9$ & 64.9 & 2100.0 & 108.0 \\
\hline 123521.651 & 0.019 & 620720.21 & 0.14 & $<2.2$ & 9.8 & 143.0 & 13.2 \\
\hline 23521.850 & 0.020 & 621224.31 & 0.14 & - & 8.8 & 116.0 & 11.7 \\
\hline 23522.229 & 0.020 & 620953.55 & 0.14 & - & 8.9 & 117.0 & 11.9 \\
\hline 123522.601 & 0.015 & 622247.91 & 0.10 & $<2.0$ & 40.4 & 1630.0 & 86.1 \\
\hline 123523.743 & 0.023 & 621648.54 & 0.16 & - & 7.2 & 95.3 & 11.5 \\
\hline 123527.444 & 0.017 & 621937.58 & 0.12 & $<2.1$ & 13.3 & 193.0 & 14.7 \\
\hline 123529.358 & 0.018 & 621256.44 & 0.13 & $<2.1$ & 11.1 & 135.0 & 11.8 \\
\hline 123531.076 & 0.025 & 621343.66 & 0.17 & - & 6.3 & 74.5 & 10.2 \\
\hline 123531.558 & 0.027 & 621117.30 & 0.19 & - & 5.7 & 66.1 & 10.1 \\
\hline 123531.749 & 0.020 & 622452.44 & 0.14 & $<2.2$ & 9.6 & 207.0 & 17.8 \\
\hline 123532.537 & 0.021 & 615517.82 & 0.15 & - & 8.0 & 358.0 & 30.8 \\
\hline 123535.036 & 0.023 & 620850.55 & 0.16 & - & 7.0 & 82.3 & 10.6 \\
\hline 123537.407 & 0.024 & 621003.13 & 0.17 & - & 6.6 & 73.5 & 10.1 \\
\hline 123537.438 & 0.021 & 620537.23 & 0.15 & - & 8.0 & 110.0 & 12.0 \\
\hline 123538.032 & 0.014 & 621932.26 & 0.10 & 5.9 & 100.7 & 6340.0 & 318.0 \\
\hline 123538.104 & 0.020 & 622240.96 & 0.14 & - & 9.1 & 149.0 & 14.2 \\
\hline 123538.267 & 0.028 & 620651.46 & 0.19 & - & 5.5 & 68.9 & 10.7 \\
\hline 123538.515 & 0.014 & 621643.07 & 0.10 & 19.7 & 78.7 & 3550.0 & 179.0 \\
\hline 123539.909 & 0.023 & 621430.66 & 0.16 & - & 7.0 & 74.9 & 9.8 \\
\hline 123539.953 & 0.023 & 621441.98 & 0.16 & - & 7.1 & 76.2 & 9.9 \\
\hline 123540.388 & 0.028 & 621623.80 & 0.19 & - & 5.4 & 60.1 & 9.8 \\
\hline 123540.998 & 0.022 & 621828.20 & 0.16 & - & 7.5 & 89.0 & 10.8 \\
\hline 123541.100 & 0.029 & 620120.42 & 0.20 & - & 5.4 & 111.0 & 14.5 \\
\hline 123541.109 & 0.021 & 620821.46 & 0.15 & 3.9 & 8.2 & 270.0 & 16.5 \\
\hline 123542.083 & 0.022 & 620535.75 & 0.16 & - & 7.6 & 100.0 & 11.6 \\
\hline 123544.177 & 0.021 & 615731.29 & 0.15 & 3.6 & 8.0 & 526.0 & 32.0 \\
\hline 123544.782 & 0.019 & 622242.06 & 0.13 & 6.6 & 10.0 & 442.0 & 24.9 \\
\hline 123545.056 & 0.022 & 615448.65 & 0.15 & - & 7.7 & 620.0 & 39.5 \\
\hline 123545.737 & 0.016 & 620240.18 & 0.11 & 2.4 & 15.9 & 318.0 & 19.9 \\
\hline 123547.105 & 0.023 & 622009.50 & 0.16 & - & 7.4 & 92.5 & 11.1 \\
\hline
\end{tabular}




\begin{tabular}{|c|c|c|c|c|c|c|c|}
\hline 123547.760 & 0.019 & $62 \quad 1057.81$ & 0.13 & $<2.2$ & 9.9 & 99.8 & 10.1 \\
\hline 123547.962 & 0.018 & 621529.42 & 0.13 & $<2.1$ & 11.7 & 118.0 & 10.6 \\
\hline 123548.101 & 0.022 & 621034.97 & 0.15 & - & 8.0 & 80.2 & 9.7 \\
\hline 123548.814 & 0.022 & 622602.24 & 0.15 & - & 7.7 & 130.0 & 15.7 \\
\hline 123549.422 & 0.022 & 621536.77 & 0.16 & - & 7.5 & 74.6 & 9.5 \\
\hline 123550.618 & 0.014 & 622758.03 & 0.10 & 4.4 & 116.7 & 11400.0 & 570.0 \\
\hline 123550.716 & 0.019 & 620721.52 & 0.13 & $<2.2$ & 10.4 & 115.0 & 11.0 \\
\hline 123550.927 & 0.019 & 621457.42 & 0.13 & $<2.1$ & 10.9 & 107.0 & 10.2 \\
\hline 123551.239 & 0.017 & 622514.79 & 0.12 & 2.4 & 15.5 & 354.0 & 22.2 \\
\hline 123551.943 & 0.020 & 620809.09 & 0.14 & $<2.2$ & 9.4 & 98.7 & 10.3 \\
\hline 123553.281 & 0.026 & 621337.85 & 0.18 & - & 6.1 & 58.4 & 9.0 \\
\hline 123553.748 & 0.024 & 622155.20 & 0.17 & - & 6.8 & 83.6 & 11.5 \\
\hline 123554.092 & 0.027 & 621043.37 & 0.19 & - & 5.6 & 53.7 & 9.0 \\
\hline 123555.144 & 0.016 & 620901.80 & 0.11 & $<2.0$ & 21.3 & 212.0 & 13.7 \\
\hline 123555.977 & 0.023 & 621555.78 & 0.16 & 2.5 & 7.3 & 89.7 & 9.7 \\
\hline 123556.380 & 0.026 & 620247.23 & 0.18 & - & 5.9 & 87.7 & 12.1 \\
\hline 123557.023 & 0.028 & 620212.23 & 0.19 & - & 5.5 & 98.7 & 12.6 \\
\hline 123557.624 & 0.028 & 620808.11 & 0.19 & - & 5.4 & 68.6 & 9.5 \\
\hline 123557.953 & 0.015 & 621536.86 & 0.11 & $<2.0$ & 22.2 & 209.0 & 13.4 \\
\hline 123559.752 & 0.017 & 621550.05 & 0.12 & 2.7 & 14.7 & 212.0 & 13.5 \\
\hline 123600.064 & 0.019 & 620253.36 & 0.13 & 2.8 & 9.9 & 262.0 & 17.1 \\
\hline 123600.164 & 0.017 & 621047.38 & 0.12 & $<2.1$ & 14.3 & 131.0 & 10.6 \\
\hline 123600.940 & 0.024 & 621807.21 & 0.17 & - & 6.5 & 65.7 & 9.4 \\
\hline 123601.576 & 0.026 & 622819.54 & 0.19 & - & 5.8 & 204.0 & 19.4 \\
\hline 123601.796 & 0.019 & 621126.42 & 0.13 & $<2.2$ & 10.3 & 93.2 & 9.5 \\
\hline 123601.987 & 0.027 & 620604.93 & 0.19 & - & 5.7 & 77.2 & 10.1 \\
\hline 123602.339 & 0.025 & 622425.73 & 0.18 & - & 6.3 & 102.0 & 13.0 \\
\hline 123603.276 & 0.017 & 621111.17 & 0.12 & $<2.1$ & 13.8 & 124.0 & 10.3 \\
\hline 123604.196 & 0.021 & 621620.34 & 0.14 & - & 8.7 & 80.6 & 9.3 \\
\hline 123604.289 & 0.027 & 620811.57 & 0.19 & - & 5.7 & 55.4 & 9.1 \\
\hline 123605.503 & 0.029 & 620158.79 & 0.20 & - & 5.4 & 90.3 & 12.3 \\
\hline 123606.484 & 0.023 & 620608.78 & 0.16 & - & 7.1 & 76.1 & 9.9 \\
\hline 123606.622 & 0.016 & 620951.33 & 0.11 & 2.2 & 19.4 & 196.0 & 12.8 \\
\hline 123606.887 & 0.021 & 621021.67 & 0.15 & - & 8.4 & 74.4 & 9.0 \\
\hline 123607.139 & 0.026 & 621328.77 & 0.18 & - & 5.8 & 51.3 & 8.4 \\
\hline 123608.137 & 0.015 & 621036.01 & 0.11 & $<2.0$ & 24.7 & 217.0 & 13.6 \\
\hline
\end{tabular}




\begin{tabular}{|c|c|c|c|c|c|c|c|}
\hline 3608.234 & 0.022 & $62 \quad 15 \quad 52.92$ & 0.15 & - & 6.6 & 59.3 & 8.6 \\
\hline 3608.582 & 0.022 & 621435.20 & 0.16 & - & 7.5 & 68.9 & 8.8 \\
\hline 3608.734 & 0.020 & 623054.30 & 0.14 & $<2.2$ & 9.6 & 419.0 & 29.8 \\
\hline 3609.430 & 0.025 & 622042.60 & 0.18 & - & 6.1 & 68.7 & 10.0 \\
\hline 3609.901 & 0.020 & 622045.97 & 0.14 & - & 8.9 & 100.0 & 10.7 \\
\hline 3610.187 & 0.027 & 620644.90 & 0.19 & - & 5.5 & 55.6 & 9.3 \\
\hline 3610.526 & 0.025 & 620811.08 & 0.17 & - & 6.3 & 59.0 & 9.0 \\
\hline 23610.583 & 0.018 & 621651.77 & 0.12 & 2.7 & 12.4 & 139.0 & 10.8 \\
\hline 23610.708 & 0.018 & 622345.24 & 0.13 & $<2.1$ & 11.9 & 173.0 & 14.1 \\
\hline 123611.103 & 0.018 & 622228.69 & 0.13 & $<2.1$ & 11.9 & 154.0 & 12.8 \\
\hline 23611.408 & 0.020 & 622149.93 & 0.14 & - & 9.1 & 111.0 & 11.4 \\
\hline 23612.223 & 0.017 & 620448.70 & 0.12 & $<2.1$ & 14.1 & 161.0 & 12.5 \\
\hline 123612.455 & 0.020 & 621140.46 & 0.14 & 3.9 & 9.7 & 174.0 & 11.8 \\
\hline 123613.618 & 0.022 & 620248.56 & 0.16 & - & 7.4 & 100.0 & 11.7 \\
\hline 123614.762 & 0.028 & 620134.00 & 0.19 & - & 5.4 & 82.4 & 12.1 \\
\hline 123614.805 & 0.022 & 615429.90 & 0.16 & - & 7.5 & 285.0 & 26.3 \\
\hline 123615.035 & 0.023 & $6206 \quad 13.21$ & 0.16 & - & 7.0 & 89.2 & 10.0 \\
\hline 123615.607 & 0.025 & 620946.74 & 0.18 & - & 5.6 & 48.8 & 8.3 \\
\hline 123616.091 & 0.027 & 622203.96 & 0.19 & - & 5.6 & 68.4 & 10.5 \\
\hline 123616.135 & 0.025 & 621513.98 & 0.17 & - & 6.4 & 53.9 & 8.4 \\
\hline 123616.524 & 0.026 & 620702.71 & 0.18 & - & 5.9 & 56.6 & 9.1 \\
\hline 123617.105 & 0.023 & 621011.57 & 0.16 & - & 7.3 & 62.0 & 8.6 \\
\hline 123617.565 & 0.015 & 621540.91 & 0.11 & $<2.0$ & 23.6 & 200.0 & 12.8 \\
\hline 123618.037 & 0.028 & 621635.69 & 0.19 & - & 5.4 & 47.1 & 8.4 \\
\hline 123618.348 & 0.016 & 621550.67 & 0.11 & $<2.0$ & 17.8 & 151.0 & 11.0 \\
\hline 123619.290 & 0.021 & 620558.94 & 0.15 & - & 8.5 & 86.7 & 9.9 \\
\hline 123619.508 & 0.020 & 621252.70 & 0.14 & 2.7 & 9.4 & 108.0 & 9.4 \\
\hline 123620.285 & 0.017 & 620844.27 & 0.12 & $<2.1$ & 14.0 & 123.0 & 10.2 \\
\hline 123620.781 & 0.024 & 622509.76 & 0.17 & - & 6.7 & 111.0 & 13.1 \\
\hline 123621.025 & 0.022 & 620713.22 & 0.15 & - & 7.6 & 90.4 & 9.6 \\
\hline 123621.098 & 0.018 & 622133.88 & 0.12 & $<2.1$ & 12.5 & 143.0 & 11.9 \\
\hline 123621.267 & 0.025 & 621109.51 & 0.17 & - & 6.3 & 52.6 & 8.2 \\
\hline 123621.271 & 0.016 & 621708.69 & 0.11 & $<2.0$ & 17.0 & 148.0 & 11.0 \\
\hline 123622.408 & 0.023 & 621544.75 & 0.16 & 2.9 & 7.0 & 83.9 & 8.9 \\
\hline 123622.514 & 0.016 & 620654.04 & 0.11 & $<2.0$ & 16.6 & 159.0 & 11.7 \\
\hline 123622.656 & 0.021 & $62 \quad 16 \quad 29.94$ & 0.15 & - & 8.3 & 70.9 & 8.7 \\
\hline
\end{tabular}




\begin{tabular}{|c|c|c|c|c|c|c|c|}
\hline 23622.718 & 0.023 & $62 \quad 0945.81$ & 0.16 & - & 6.1 & 51.0 & .2 \\
\hline 23623.350 & 0.023 & 620605.29 & 0.16 & - & 7.2 & 71.7 & 9.5 \\
\hline 123623.406 & 0.025 & 620334.28 & 0.17 & - & 6.3 & 76.8 & 10.6 \\
\hline 23623.550 & 0.014 & 621642.90 & 0.10 & $<1.9$ & 56.3 & 481.0 & 25.4 \\
\hline 3623.914 & 0.025 & 620330.81 & 0.18 & - & 6.1 & 75.1 & 10.6 \\
\hline 3624.267 & 0.024 & 621017.33 & 0.17 & - & 6.5 & 54.2 & 8.3 \\
\hline 23624.298 & 0.024 & 622420.82 & 0.17 & - & 6.8 & 87.9 & 12.0 \\
\hline 123624.756 & 0.020 & 621743.88 & 0.14 & - & 8.9 & 78.8 & 9.1 \\
\hline 123628.906 & 0.026 & 620616.00 & 0.18 & - & 6.0 & 58.1 & 9.1 \\
\hline 123629.019 & 0.022 & 621046.03 & 0.15 & - & 7.8 & 81.4 & 8.7 \\
\hline 123629.245 & 0.016 & 620253.94 & 0.11 & $<2.0$ & 16.9 & 216.0 & 14.8 \\
\hline 123629.968 & 0.021 & 620542.21 & 0.15 & - & 8.2 & 82.9 & 9.7 \\
\hline 123630.094 & 0.026 & 620923.59 & 0.18 & - & 5.6 & 46.3 & 8.2 \\
\hline 123630.463 & 0.025 & 620851.03 & 0.18 & - & 5.7 & 48.0 & 8.2 \\
\hline 123631.197 & 0.015 & 622805.98 & 0.11 & 3.6 & 23.1 & 1230.0 & 63.4 \\
\hline 123631.252 & 0.017 & 620957.99 & 0.12 & 2.5 & 15.3 & 152.0 & 10.9 \\
\hline 123632.356 & 0.020 & 621700.06 & 0.14 & - & 8.8 & 81.8 & 8.9 \\
\hline 123632.579 & 0.023 & 620800.22 & 0.16 & & & 00.6 & 9.3 \\
\hline 123632.796 & 0.020 & 622420.34 & 0.14 & $<2.2$ & 9.4 & 139.0 & 13.0 \\
\hline 123633.531 & 0.016 & 622145.02 & 0.11 & $<2.0$ & 20.3 & 230.0 & 14.9 \\
\hline 123633.666 & 0.021 & 620445.37 & 0.14 & - & 8.7 & 93.6 & 10.3 \\
\hline 123633.746 & 0.027 & 621006.23 & 0.19 & - & 5.7 & 46.5 & 8.1 \\
\hline 123634.197 & 0.023 & 622040.70 & 0.16 & - & 7.0 & 72.6 & 9.6 \\
\hline 123634.454 & 0.028 & 615534.37 & 0.19 & - & 5.4 & 167.0 & 20.4 \\
\hline 123634.464 & 0.015 & 621213.33 & 0.11 & 2.3 & 23.5 & 233.0 & 13.9 \\
\hline 123634.530 & 0.016 & 621241.23 & 0.11 & 2.5 & 20.8 & 230.0 & 13.8 \\
\hline 123634.635 & 0.019 & 621926.33 & 0.13 & $<2.2$ & 10.4 & 98.8 & 9.8 \\
\hline 123634.888 & 0.023 & 621923.69 & 0.16 & - & 7.2 & 68.3 & 9.1 \\
\hline 123635.618 & 0.022 & 621424.33 & 0.16 & 2.8 & 7.5 & 87.8 & 8.8 \\
\hline 123636.335 & 0.027 & 620706.81 & 0.19 & - & 5.4 & 49.9 & 8.5 \\
\hline 123636.926 & 0.022 & 621320.36 & 0.15 & - & 6.6 & 50.0 & 7.9 \\
\hline 123637.041 & 0.021 & 620852.58 & 0.15 & - & 8.4 & 71.1 & 8.7 \\
\hline 123638.985 & 0.020 & 620230.77 & 0.14 & 2.5 & 9.0 & 166.0 & 13.2 \\
\hline 123640.151 & 0.014 & 622037.38 & 0.10 & $<1.9$ & 91.3 & 1840.0 & 93.8 \\
\hline 123640.567 & 0.015 & 621833.17 & 0.10 & $<2.0$ & 35.9 & 324.0 & 18.2 \\
\hline 123640.728 & 0.026 & 621010.85 & 0.18 & 3.7 & 5.9 & 86.8 & 8.8 \\
\hline
\end{tabular}




\begin{tabular}{|c|c|c|c|c|c|c|c|}
\hline 123641.554 & 0.020 & 620948.63 & 0.14 & - & 9.1 & 75.8 & 8.6 \\
\hline 123642.109 & 0.015 & 621331.73 & 0.10 & 2.2 & 51.2 & 467.0 & 24.6 \\
\hline 123642.161 & 0.028 & 622438.89 & 0.20 & - & 5.4 & 82.1 & 11.8 \\
\hline 123642.229 & 0.017 & 621545.73 & 0.12 & 2.4 & 14.5 & 150.0 & 10.7 \\
\hline 123643.642 & 0.018 & 622511.73 & 0.12 & $<2.1$ & 12.3 & 202.0 & 15.4 \\
\hline 123643.847 & 0.029 & 620559.53 & 0.20 & - & 5.4 & 62.5 & 9.2 \\
\hline 123644.369 & 0.014 & 621133.19 & 0.10 & 12.0 & 55.2 & 1290.0 & 61.2 \\
\hline 123644.503 & 0.020 & 620120.80 & 0.14 & - & 9.3 & 143.0 & 13.2 \\
\hline 123645.864 & 0.027 & 620754.45 & 0.19 & - & 5.5 & 48.9 & 8.4 \\
\hline 123646.047 & 0.017 & 621448.85 & 0.12 & 2.3 & 12.9 & 124.0 & 9.8 \\
\hline 123646.337 & 0.015 & 621629.77 & 0.10 & 2.7 & 28.5 & 393.0 & 21.1 \\
\hline 123646.362 & 0.016 & 621404.98 & 0.11 & $<2.0$ & 21.1 & 179.0 & 11.7 \\
\hline 123646.710 & 0.020 & 620833.62 & 0.14 & - & 9.2 & 80.1 & 8.9 \\
\hline 123646.736 & 0.027 & 621226.93 & 0.19 & - & 5.5 & 72.0 & 9.1 \\
\hline 123646.755 & 0.024 & 621445.88 & 0.16 & 3.1 & 6.9 & 117.0 & 9.6 \\
\hline 123648.461 & 0.025 & 622424.92 & 0.18 & - & 6.1 & 92.4 & 11.8 \\
\hline 123649.001 & 0.015 & 620438.80 & 0.10 & $<2.0$ & 36.0 & 850.0 & 46.3 \\
\hline 123649.165 & 0.017 & 615930.90 & 0.12 & $<2.1$ & 13.3 & 264.0 & 18.4 \\
\hline 123649.499 & 0.018 & 622143.18 & 0.12 & $<2.1$ & 12.0 & 138.0 & 11.6 \\
\hline 123649.656 & 0.027 & 621313.16 & 0.19 & - & 5.6 & 49.2 & 7.9 \\
\hline 123649.663 & 0.015 & 620738.18 & 0.10 & $<2.0$ & 33.8 & 307.0 & 17.4 \\
\hline 123650.047 & 0.026 & 620801.91 & 0.18 & - & 6.0 & 53.8 & 8.5 \\
\hline 123650.205 & 0.020 & 620844.72 & 0.14 & - & 8.8 & 76.4 & 8.8 \\
\hline 123650.208 & 0.019 & 622732.37 & 0.13 & 4.4 & 10.5 & 660.0 & 35.8 \\
\hline 123651.052 & 0.026 & 620529.32 & 0.18 & - & 5.9 & 60.3 & 9.3 \\
\hline 123651.148 & 0.018 & 621030.95 & 0.13 & $<2.1$ & 11.3 & 95.0 & 9.0 \\
\hline 123651.498 & 0.023 & 620644.03 & 0.16 & - & 7.2 & 68.5 & 9.0 \\
\hline 123651.701 & 0.028 & 620502.68 & 0.19 & - & 5.4 & 57.6 & 9.4 \\
\hline 123651.797 & 0.027 & 621221.42 & 0.19 & - & 5.7 & 49.3 & 7.9 \\
\hline 123652.354 & 0.026 & 620206.30 & 0.18 & - & 6.1 & 85.2 & 11.3 \\
\hline 123652.814 & 0.021 & 621807.40 & 0.15 & 3.7 & 8.1 & 350.0 & 23.8 \\
\hline 123652.955 & 0.016 & 621444.34 & 0.11 & 2.5 & 15.9 & 168.0 & 11.3 \\
\hline 123653.431 & 0.022 & 621140.10 & 0.15 & - & 7.8 & 65.7 & 8.2 \\
\hline 123654.303 & 0.023 & 620745.62 & 0.16 & - & 7.1 & 63.2 & 8.7 \\
\hline 123654.708 & 0.023 & 621039.57 & 0.16 & - & 6.2 & 48.2 & 8.0 \\
\hline 123654.786 & 0.028 & 620426.87 & 0.19 & - & 5.5 & 60.5 & 9.7 \\
\hline
\end{tabular}




\begin{tabular}{|c|c|c|c|c|c|c|c|}
\hline 123654.859 & 0.024 & 620535.22 & 0.17 & 2.7 & 6.8 & 110.0 & 10.3 \\
\hline 123655.768 & 0.025 & 615648.08 & 0.17 & 3.6 & 6.4 & 312.0 & 22.5 \\
\hline 123655.785 & 0.022 & 620917.66 & 0.15 & - & 7.6 & 64.2 & 8.4 \\
\hline 123655.837 & 0.014 & 615659.39 & 0.10 & 37.2 & 83.3 & 36900.0 & 1850.0 \\
\hline 123655.946 & 0.018 & 620808.43 & 0.12 & $<2.1$ & 12.1 & 106.0 & 9.6 \\
\hline 123656.126 & 0.025 & 615644.46 & 0.18 & 5.0 & 6.1 & 552.0 & 32.1 \\
\hline 123656.174 & 0.028 & 622631.39 & 0.20 & - & 5.4 & 106.0 & 13.8 \\
\hline 123656.505 & 0.027 & 621938.07 & 0.19 & - & 5.5 & 53.3 & 8.9 \\
\hline 123656.605 & 0.027 & 621207.58 & 0.19 & - & 5.5 & 46.2 & 7.9 \\
\hline 123656.884 & 0.027 & 621302.52 & 0.19 & - & 5.7 & 49.5 & 7.9 \\
\hline 123657.858 & 0.024 & 622332.53 & 0.17 & - & 6.6 & 89.2 & 11.2 \\
\hline 123659.356 & 0.015 & 621832.58 & 0.10 & $<2.0$ & 29.8 & 506.0 & 30.2 \\
\hline 123659.617 & 0.029 & 622509.94 & 0.20 & - & 5.5 & 90.3 & 12.4 \\
\hline 123659.948 & 0.023 & 621449.87 & 0.16 & - & 6.1 & 47.0 & 7.9 \\
\hline 123700.263 & 0.015 & 620909.99 & 0.10 & $<2.0$ & 38.5 & 324.0 & 18.0 \\
\hline 123701.104 & 0.015 & 622109.68 & 0.10 & $<2.0$ & 28.3 & 304.0 & 17.7 \\
\hline 123701.548 & 0.025 & 622025.07 & 0.18 & - & 6.3 & 63.5 & 9.4 \\
\hline 123701.560 & 0.020 & 621146.86 & 0.14 & 3.0 & 9.7 & 128.0 & 9.9 \\
\hline 123702.123 & 0.029 & 622330.56 & 0.20 & - & 5.5 & 78.7 & 11.1 \\
\hline 123702.672 & 0.027 & 623045.38 & 0.19 & - & 5.5 & 188.0 & 21.6 \\
\hline 123702.762 & 0.026 & 621401.61 & 0.18 & - & 5.4 & 41.4 & 7.8 \\
\hline 123704.077 & 0.025 & 620755.54 & 0.17 & - & 5.8 & 50.7 & 8.4 \\
\hline 123705.211 & 0.024 & 621950.89 & 0.17 & - & 6.7 & 65.9 & 9.2 \\
\hline 123705.875 & 0.025 & 621153.66 & 0.17 & - & 6.4 & 52.5 & 8.0 \\
\hline 123706.769 & 0.021 & 620722.42 & 0.15 & - & 8.0 & 72.8 & 9.0 \\
\hline 123706.893 & 0.019 & 622306.53 & 0.13 & 2.5 & 10.5 & 189.0 & 13.9 \\
\hline 123707.203 & 0.027 & 621408.33 & 0.19 & - & 5.5 & 45.3 & 7.9 \\
\hline 123707.521 & 0.025 & 622148.24 & 0.18 & - & 6.1 & 70.0 & 10.1 \\
\hline 123707.925 & 0.019 & 620545.21 & 0.14 & $<2.2$ & 9.8 & 98.0 & 10.1 \\
\hline 123707.959 & 0.023 & 621121.81 & 0.16 & - & 7.4 & 60.3 & 8.2 \\
\hline 123708.114 & 0.026 & 621659.30 & 0.18 & - & 5.9 & 50.4 & 8.3 \\
\hline 123708.335 & 0.027 & 621056.23 & 0.19 & - & 5.5 & 45.1 & 8.0 \\
\hline 123708.786 & 0.017 & 622201.91 & 0.12 & $<2.1$ & 14.6 & 170.0 & 12.8 \\
\hline 123709.056 & 0.021 & 622319.20 & 0.14 & - & 8.6 & 114.0 & 11.8 \\
\hline 123709.449 & 0.021 & 620837.83 & 0.15 & - & 8.4 & 72.0 & 8.8 \\
\hline 123709.769 & 0.020 & 620841.22 & 0.14 & - & 7.9 & 67.9 & 8.3 \\
\hline
\end{tabular}




\begin{tabular}{|c|c|c|c|c|c|c|c|}
\hline 123709.941 & 0.014 & 622258.87 & 0.10 & $<1.9$ & 55.5 & 708.0 & 36.9 \\
\hline 3710.018 & 0.021 & 622650.25 & 0.15 & 5.9 & 8.1 & 551.0 & 30.6 \\
\hline 3711.339 & 0.019 & 621331.26 & 0.13 & $<2.1$ & 10.8 & 132.0 & 10.1 \\
\hline 3711.946 & 0.025 & 621325.89 & 0.17 & - & 6.5 & 53.9 & 8.1 \\
\hline 23712.530 & 0.024 & 615706.75 & 0.16 & 3.4 & 6.9 & 368.0 & 24.4 \\
\hline 123712.555 & 0.024 & 622425.02 & 0.17 & - & 6.7 & 100.0 & 12.2 \\
\hline 123713.582 & 0.029 & 621604.00 & 0.20 & - & 5.5 & 55.3 & 8.3 \\
\hline 123713.870 & 0.014 & 621826.47 & 0.10 & $<1.9$ & 64.9 & 595.0 & 30.9 \\
\hline 123714.336 & 0.028 & 620504.15 & 0.19 & - & 5.5 & 58.6 & 9.6 \\
\hline 123714.964 & 0.014 & 620823.45 & 0.10 & $<1.9$ & 79.3 & 1380.0 & 70.8 \\
\hline 123715.618 & 0.027 & 620108.51 & 0.19 & 3.4 & 5.7 & 145.0 & 13.6 \\
\hline 123716.032 & 0.020 & 620323.04 & 0.14 & - & 8.8 & 109.0 & 11.4 \\
\hline 123716.380 & 0.015 & 621512.61 & 0.11 & $<2.0$ & 22.5 & 187.0 & 12.2 \\
\hline 123716.530 & 0.025 & 620210.06 & 0.18 & - & 6.1 & 85.2 & 11.6 \\
\hline 123716.560 & 0.022 & 621643.94 & 0.15 & - & 7.8 & 66.5 & 8.7 \\
\hline 123716.681 & 0.015 & 621733.53 & 0.10 & $<2.0$ & 39.0 & 346.0 & 19.2 \\
\hline 123716.787 & 0.022 & 621007.45 & 0.16 & - & 7.6 & 63.3 & 8.5 \\
\hline 123717.511 & 0.023 & $6208 \quad 27.43$ & 0.16 & 2.1 & 7.1 & 126.0 & 17.5 \\
\hline 123717.608 & 0.024 & 615645.38 & 0.17 & 3.0 & 6.6 & 311.0 & 22.9 \\
\hline 123718.578 & 0.025 & 620336.37 & 0.18 & - & 6.3 & 76.7 & 10.6 \\
\hline 123718.716 & 0.014 & 620355.81 & 0.10 & $<1.9$ & 282.0 & 6580.0 & 330.0 \\
\hline 123720.019 & 0.018 & 620741.45 & 0.13 & 2.2 & 11.5 & 202.0 & 19.6 \\
\hline 123721.268 & 0.016 & 621130.17 & 0.11 & 0.9 & 20.7 & 382.0 & 24.7 \\
\hline 123721.393 & 0.015 & 620708.47 & 0.10 & $<2.0$ & 32.5 & 310.0 & 17.7 \\
\hline 123721.412 & 0.026 & 621346.79 & 0.18 & - & 6.0 & 50.5 & 8.2 \\
\hline 123722.301 & 0.027 & 622751.84 & 0.19 & 3.5 & 5.6 & 258.0 & 19.8 \\
\hline 123723.057 & 0.022 & 620539.87 & 0.16 & - & 7.4 & 78.5 & 9.9 \\
\hline 123725.045 & 0.019 & 620856.67 & 0.13 & $<2.2$ & 10.0 & 90.0 & 9.4 \\
\hline 123725.294 & 0.025 & 621006.51 & 0.17 & 3.9 & 6.4 & 117.0 & 10.0 \\
\hline 123725.679 & 0.028 & 621941.40 & 0.19 & 3.4 & 5.4 & 122.0 & 10.8 \\
\hline 123725.726 & 0.014 & 621128.50 & 0.10 & 5.5 & 98.0 & 5960.0 & 298.0 \\
\hline 123726.370 & 0.016 & 615817.94 & 0.11 & 3.6 & 17.0 & 883.0 & 46.6 \\
\hline 123726.476 & 0.020 & 622026.77 & 0.14 & $<2.2$ & 9.4 & 102.0 & 10.6 \\
\hline 123730.799 & 0.018 & $6212 \quad 59.14$ & 0.12 & $<2.1$ & 12.0 & 107.0 & 9.6 \\
\hline 123731.141 & 0.025 & 623137.19 & 0.17 & 2.8 & 6.4 & 369.0 & 29.7 \\
\hline 123732.642 & 0.026 & $62 \quad 1012.87$ & 0.18 & - & 5.6 & 50.6 & 8.5 \\
\hline
\end{tabular}




\begin{tabular}{|c|c|c|c|c|c|c|c|}
\hline 123734.274 & 0.020 & 620931.86 & 0.14 & 2.8 & 9.7 & 142.0 & 11.0 \\
\hline 123734.513 & 0.020 & 621723.29 & 0.14 & 2.7 & 9.5 & 131.0 & 10.8 \\
\hline 3735.147 & 0.023 & 622559.88 & 0.16 & 4.0 & 7.1 & 381.0 & 23.2 \\
\hline 3735.403 & 0.026 & 620941.49 & 0.18 & - & 5.9 & 54.8 & 8.8 \\
\hline 3735.869 & 0.029 & 622402.78 & 0.20 & - & 5.5 & 93.1 & 12.5 \\
\hline 23736.502 & 0.022 & 620609.50 & 0.16 & - & 7.5 & 81.9 & 10.2 \\
\hline 23736.586 & 0.024 & 615919.13 & 0.17 & 3.2 & 6.5 & 248.0 & 18.8 \\
\hline 3736.879 & 0.025 & 621429.40 & 0.17 & - & 6.4 & 57.8 & 8.7 \\
\hline 123737.111 & 0.027 & 621205.40 & 0.19 & 3.4 & 5.7 & 100.0 & 9.6 \\
\hline 23738.118 & 0.017 & 620828.12 & 0.12 & $<2.1$ & 13.8 & 135.0 & 11.0 \\
\hline 23738.221 & 0.022 & 620920.46 & 0.16 & - & 7.5 & 71.9 & 9.3 \\
\hline 123739.279 & 0.026 & 622059.62 & 0.18 & - & 6.0 & 72.5 & 10.5 \\
\hline 123739.329 & 0.015 & 620505.64 & 0.11 & $<2.0$ & 25.8 & 309.0 & 18.3 \\
\hline 123740.823 & 0.027 & 621231.23 & 0.19 & 3.7 & 5.5 & 91.0 & 9.5 \\
\hline 123740.898 & 0.027 & 620444.10 & 0.19 & - & 5.7 & 71.0 & 10.7 \\
\hline 123741.028 & 0.022 & 620911.01 & 0.15 & - & 7.7 & 74.6 & 9.4 \\
\hline 123741.394 & 0.016 & 621251.43 & 0.11 & $<2.0$ & 17.3 & 163.0 & 11.7 \\
\hline 123742.825 & 0.023 & 621557.48 & 0.16 & - & 7.0 & 67.1 & 9.2 \\
\hline 123744.672 & 0.023 & 621219.00 & 0.16 & - & 7.0 & 67.3 & 9.1 \\
\hline 123744.674 & 0.023 & 622306.80 & 0.16 & 2.9 & 7.3 & 184.0 & 14.6 \\
\hline 123744.751 & 0.024 & 622303.87 & 0.17 & - & 6.8 & 102.0 & 12.4 \\
\hline 123745.729 & 0.024 & 621456.43 & 0.17 & - & 6.6 & 63.6 & 9.1 \\
\hline 123745.734 & 0.027 & 620429.36 & 0.19 & - & 5.6 & 73.7 & 11.0 \\
\hline 123746.641 & 0.015 & 621738.46 & 0.10 & $<1.9$ & 51.5 & 998.0 & 53.2 \\
\hline 123747.084 & 0.020 & 621631.77 & 0.14 & $<2.2$ & 9.6 & 96.9 & 10.1 \\
\hline 123747.544 & 0.024 & 622249.86 & 0.17 & - & 6.9 & 102.0 & 12.4 \\
\hline 123747.940 & 0.024 & 621442.20 & 0.17 & - & 6.6 & 64.4 & 9.2 \\
\hline 123750.262 & 0.020 & 621359.16 & 0.14 & - & 9.1 & 90.5 & 9.8 \\
\hline 123750.491 & 0.022 & 621143.33 & 0.16 & 4.5 & 7.5 & 198.0 & 13.2 \\
\hline 123751.217 & 0.016 & 621919.11 & 0.11 & $<2.0$ & 18.8 & 223.0 & 14.8 \\
\hline 123751.428 & 0.019 & 622259.70 & 0.13 & $<2.2$ & 10.1 & 157.0 & 14.0 \\
\hline 123752.699 & 0.022 & 620719.40 & 0.16 & - & 7.5 & 86.6 & 10.5 \\
\hline 123752.713 & 0.025 & 621627.95 & 0.18 & - & 6.1 & 79.8 & 9.9 \\
\hline 123752.845 & 0.017 & 622145.04 & 0.12 & $<2.1$ & 13.6 & 193.0 & 14.6 \\
\hline 123753.347 & 0.019 & 622219.79 & 0.14 & 2.8 & 9.7 & 219.0 & 15.7 \\
\hline 123754.239 & 0.024 & 621340.05 & 0.17 & - & 6.5 & 67.0 & 9.4 \\
\hline
\end{tabular}




\begin{tabular}{|c|c|c|c|c|c|c|c|}
\hline 123754.340 & 0.015 & $\begin{array}{llll}62 & 10 & 59.51\end{array}$ & 0.11 & $<2.0$ & 27.6 & 285.0 & 16.8 \\
\hline 23755.457 & 0.025 & 621142.12 & 0.17 & - & 6.4 & 66.1 & 9.5 \\
\hline 123755.726 & 0.025 & 621507.18 & 0.18 & 3.4 & 6.2 & 120.0 & 10.8 \\
\hline 123756.986 & 0.016 & 622059.32 & 0.11 & $<2.1$ & 16.0 & 221.0 & 15.4 \\
\hline 123759.523 & 0.016 & 620626.62 & 0.11 & $<2.0$ & 20.7 & 266.0 & 16.8 \\
\hline 123759.589 & 0.022 & 621102.47 & 0.15 & - & 7.9 & 85.0 & 10.1 \\
\hline 23800.927 & 0.016 & 621336.18 & 0.11 & $<2.0$ & 17.3 & 190.0 & 13.2 \\
\hline 123801.315 & 0.023 & 621332.08 & 0.16 & - & 7.2 & 79.7 & 10.0 \\
\hline 123801.434 & 0.025 & 621146.03 & 0.18 & - & 6.2 & 68.0 & 9.8 \\
\hline 123801.754 & 0.018 & 622147.85 & 0.12 & $<2.1$ & 12.5 & 191.0 & 15.0 \\
\hline 123803.649 & 0.018 & $6217 \quad 11.52$ & 0.12 & 3.0 & 12.5 & 253.0 & 16.0 \\
\hline 123805.526 & 0.023 & 621445.92 & 0.16 & - & 7.3 & 83.2 & 10.3 \\
\hline 123806.409 & 0.020 & 620324.81 & 0.14 & - & 8.8 & 150.0 & 14.5 \\
\hline 123806.510 & 0.018 & 615709.23 & 0.12 & 2.8 & 12.6 & 610.0 & 36.7 \\
\hline 123806.791 & 0.015 & 621242.25 & 0.10 & $<2.0$ & 44.3 & 1010.0 & 53.8 \\
\hline 123807.251 & 0.021 & 620718.83 & 0.15 & - & 8.2 & 107.0 & 11.7 \\
\hline 123807.447 & 0.016 & 622519.60 & 0.12 & 2.7 & 15.8 & 492.0 & 28.9 \\
\hline 123807.738 & 0.027 & 622549.78 & 0.19 & - & 5.7 & 148.0 & 17.3 \\
\hline 123808.452 & 0.027 & 622531.57 & 0.19 & - & 5.8 & 136.0 & 16.8 \\
\hline 123811.510 & 0.024 & 620942.83 & 0.17 & - & 6.7 & 83.4 & 10.9 \\
\hline 123812.507 & 0.026 & 621455.15 & 0.18 & 3.7 & 5.9 & 148.0 & 12.4 \\
\hline 123818.203 & 0.022 & 620858.27 & 0.15 & - & 7.7 & 106.0 & 11.9 \\
\hline 123818.691 & 0.027 & 621029.00 & 0.19 & - & 5.5 & 73.7 & 11.0 \\
\hline 123820.466 & 0.015 & 621828.55 & 0.11 & 6.9 & 26.1 & 1800.0 & 93.0 \\
\hline 123821.778 & 0.022 & 621706.43 & 0.15 & 2.4 & 7.9 & 152.0 & 13.4 \\
\hline 123823.765 & 0.020 & 620707.62 & 0.14 & - & 9.3 & 148.0 & 13.9 \\
\hline 123825.712 & 0.016 & 622813.73 & 0.11 & 2.7 & 17.7 & 876.0 & 49.3 \\
\hline 123826.205 & 0.024 & 615803.14 & 0.17 & - & 6.8 & 259.0 & 25.3 \\
\hline 123828.237 & 0.028 & 621823.73 & 0.20 & - & 5.2 & 98.3 & 13.0 \\
\hline 123828.544 & 0.019 & 620736.58 & 0.13 & $<2.2$ & 10.0 & 165.0 & 14.6 \\
\hline 123829.392 & 0.018 & 621649.28 & 0.12 & $<2.1$ & 12.3 & 193.0 & 15.1 \\
\hline 123829.863 & 0.024 & 621016.40 & 0.17 & - & 6.8 & 105.0 & 12.6 \\
\hline 123830.192 & 0.016 & 621401.15 & 0.11 & 2.4 & 20.4 & 368.0 & 21.6 \\
\hline 123830.630 & 0.015 & 621821.30 & 0.11 & 2.9 & 26.8 & 601.0 & 32.5 \\
\hline 123830.763 & 0.027 & 622317.61 & 0.19 & - & 5.6 & 134.0 & 16.9 \\
\hline 123837.038 & 0.021 & 620356.12 & 0.15 & 3.3 & 8.3 & 304.0 & 21.5 \\
\hline
\end{tabular}




\begin{tabular}{|c|c|c|c|c|c|c|c|}
\hline 123841.896 & 0.024 & 622234.68 & 0.16 & 5.2 & 6.9 & 357.0 & 24.3 \\
\hline 123854.957 & 0.028 & 621138.26 & 0.19 & - & 5.4 & 159.0 & 16.3 \\
\hline 123855.385 & 0.027 & 621232.38 & 0.19 & 3.7 & 5.7 & 278.0 & 19.9 \\
\hline 123859.502 & 0.026 & 620542.77 & 0.19 & - & 5.8 & 216.0 & 20.6 \\
\hline 123902.591 & 0.015 & 620211.54 & 0.11 & $<2.0$ & 26.9 & 1410.0 & 74.0 \\
\hline 3907.411 & 0.022 & 621540.66 & 0.15 & 5.7 & 7.9 & 557.0 & 32.4 \\
\hline 123911.741 & 0.017 & 622217.41 & 0.12 & 2.7 & 13.9 & 963.0 & 53.3 \\
\hline 123914.388 & 0.034 & 620859.80 & 0.24 & - & 5.5 & 171.0 & 20.5 \\
\hline 123914.969 & 0.018 & 621458.17 & 0.13 & 3.4 & 11.2 & 475.0 & 29.9 \\
\hline 123921.841 & 0.022 & 621600.19 & 0.15 & - & 7.8 & 149.0 & 21.5 \\
\hline 3927.465 & 0.027 & 620658.72 & 0.19 & - & 5.5 & 309.0 & 28.5 \\
\hline 3931.546 & 0.016 & 621555.90 & 0.11 & 2.6 & 16.2 & 807.0 & 46.6 \\
\hline
\end{tabular}




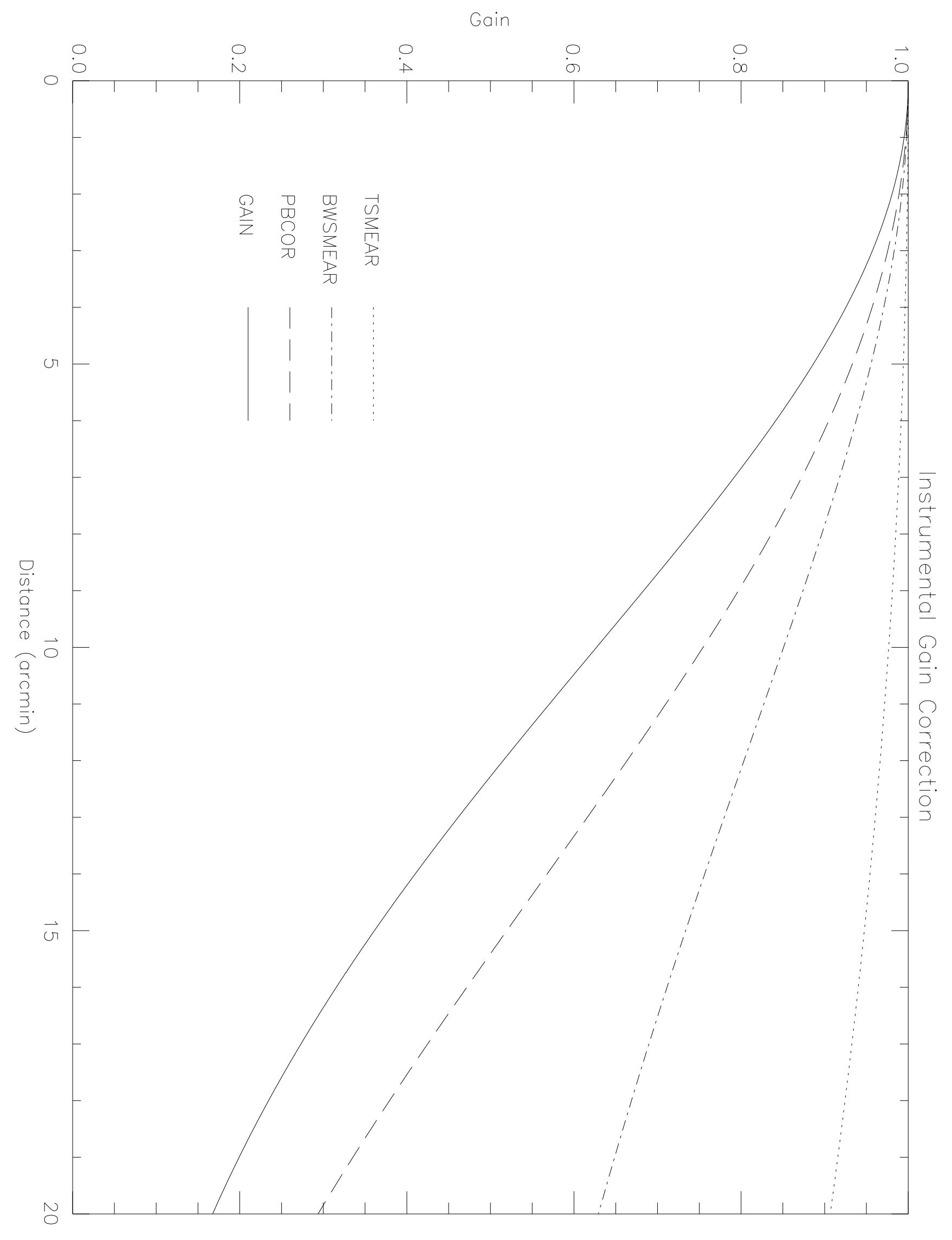


Table 3

1.4 GHz Source Count

\begin{tabular}{cccccc}
\hline \hline $\begin{array}{c}\text { Bin } \\
\mu \mathrm{Jy}\end{array}$ & $\begin{array}{c}<S_{1.4}> \\
\mu \mathrm{Jy}\end{array}$ & $\begin{array}{c}\text { Area } \\
\operatorname{arcmin}^{2}\end{array}$ & $\begin{array}{c}\mathrm{dN} / \mathrm{dS} \\
\text { ster }^{-1} \mathrm{Jy}^{-1}\end{array}$ & $\begin{array}{c}\text { Corr. } \\
\text { Factor }\end{array}$ & $\mathrm{n} / \mathrm{n}_{\circ}$ \\
\hline \hline $40-67$ & 55.4 & 232 & $9.4 \times 10^{10}$ & $1.3 \pm 0.1$ & $1.6 \pm 0.4$ \\
$67-82$ & 73.7 & 421 & $9.3 \times 10^{10}$ & $1.1 \pm 0.1$ & $3.2 \pm 0.5$ \\
$82-102$ & 91.4 & 519 & $5.7 \times 10^{10}$ & 1.0 & $3.0 \pm 0.4$ \\
$102-138$ & 116 & 654 & $2.5 \times 10^{10}$ & 1.0 & $2.6 \pm 0.4$ \\
$139-203$ & 162 & 872 & $1.1 \times 10^{10}$ & 1.0 & $2.6 \pm 0.4$ \\
$204-354$ & 249 & 1067 & $3.7 \times 10^{9}$ & 1.0 & $2.8 \pm 0.4$ \\
$357-2100$ & 512 & 1120 & $3.0 \times 10^{8}$ & 1.0 & $3.8 \pm 0.5$ \\
\hline \hline
\end{tabular}




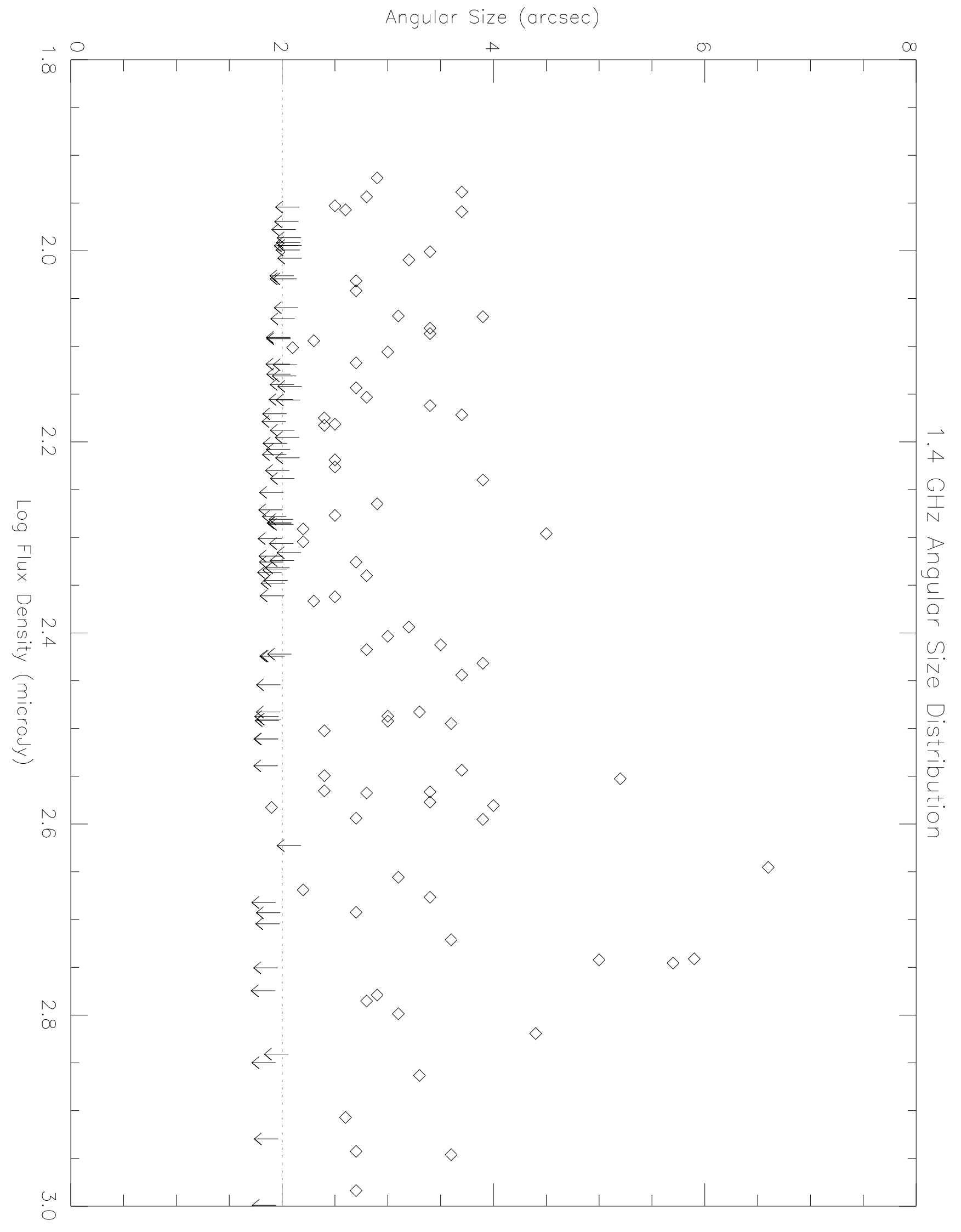


Table 4

\begin{tabular}{ccc}
\multicolumn{3}{c}{ Angular Correlation Function } \\
\hline \hline $\begin{array}{c}\text { Bin } \\
\text { arcmin }\end{array}$ & $w(\theta)$ & $\mathrm{N}_{D D}$ \\
\hline \hline $0.0-0.1$ & $-0.42 \pm 0.28$ & 12 \\
$0.1-0.3$ & $-0.27 \pm 0.17$ & 21 \\
$0.3-0.8$ & $0.12 \pm 0.17$ & 166 \\
$0.8-1.8$ & $0.25 \pm 0.05$ & 804 \\
$1.8-3.8$ & $0.22 \pm 0.06$ & 3236 \\
$3.8-7.8$ & $0.19 \pm 0.05$ & 11705 \\
$7.8-16$ & $0.06 \pm 0.06$ & 29505 \\
$16-40$ & $-0.15 \pm 0.03$ & 23186 \\
\hline \hline
\end{tabular}




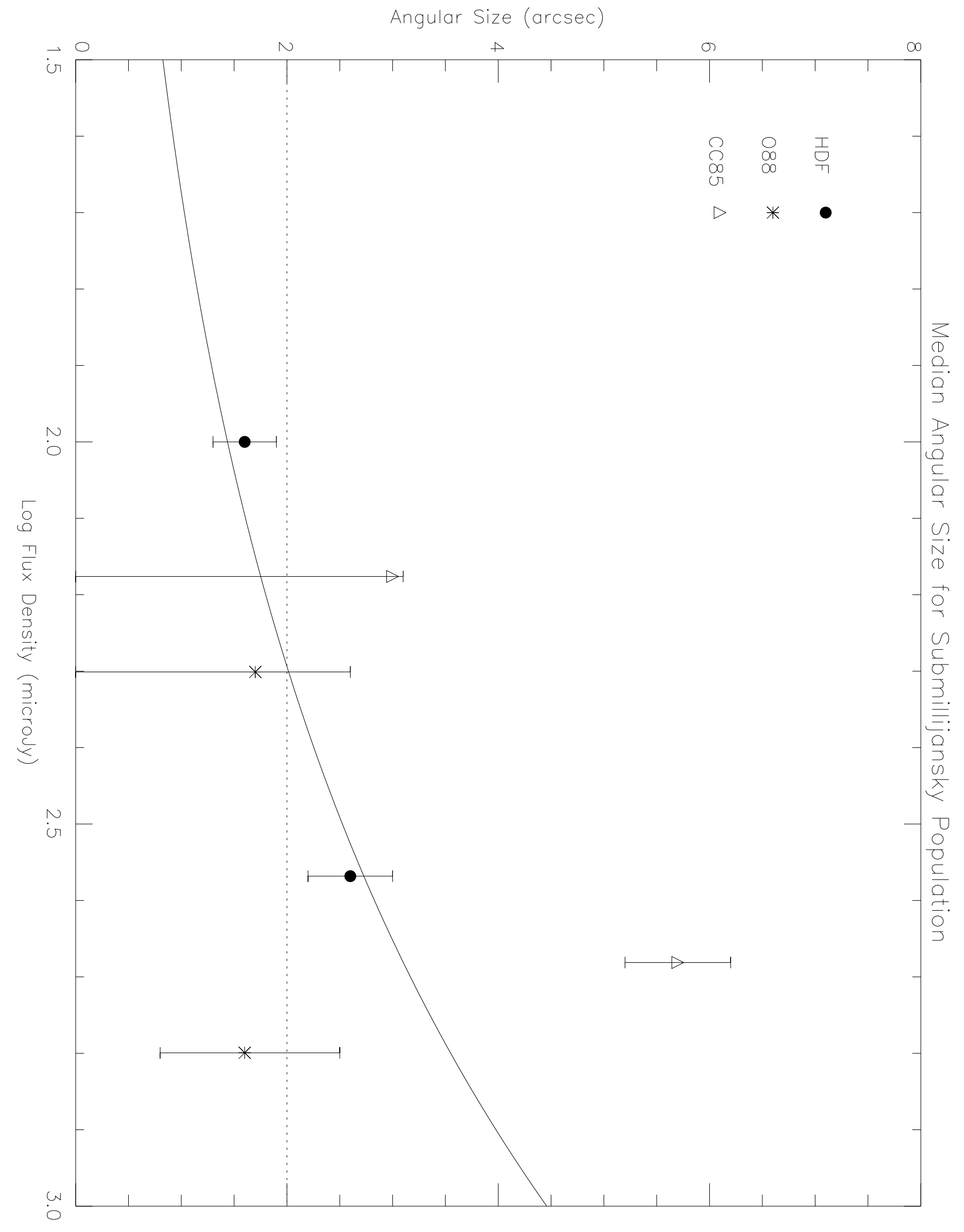



Table 5: Spectral Indices for HDF Radio Sample

\begin{tabular}{|c|c|c|c|c|c|}
\hline Source & $S_{8.5}$ & $S_{1.4}$ & $\alpha$ & $\theta_{\text {las }}$ & sample \\
\hline VLAJ123600+621047 & $<51.0$ & 125.2 & $>0.50$ & $<2.1$ & 1 \\
\hline VLAJ123606+620951 & $<70.4$ & 191.0 & $>0.56$ & 1.9 & 1 \\
\hline VLAJ123608+621035 & 123.0 & 233.0 & $0.36 \pm 0.08$ & $<2.0$ & $\mathrm{~h}, \mathrm{l}$ \\
\hline VLAJ123608+621552 & 90.5 & 52.9 & $-0.24 \pm 0.10$ & - & $\mathrm{h}, \mathrm{l}$ \\
\hline VLAJ123612+621140 & $<24.0$ & 119.0 & $>0.89$ & 2.4 & 1 \\
\hline VLAJ123618+621540 & $<71.4$ & 190.0 & $>0.55$ & $<2.0$ & 1 \\
\hline VLAJ123618+621550 & $<47.0$ & 145.0 & $>0.63$ & $<2.0$ & 1 \\
\hline VLAJ123619+621252 & $<21.8$ & 91.4 & $>0.80$ & 2.0 & 1 \\
\hline VLAJ123620+620844 & 218.00 & 220.0 & $0.01 \pm 0.07$ & $<2.1$ & $\mathrm{~h}, \mathrm{l}$ \\
\hline VLAJ123621+621108 & $<15.0$ & 69.6 & $>0.86$ & - & 1 \\
\hline VLAJ123621+621708 & $<58.7$ & 159.0 & $>0.56$ & $<2.0$ & 1 \\
\hline VLAJ123622+621544 & $<24.6$ & 72.5 & $>0.60$ & 2.0 & 1 \\
\hline VLAJ123623+621642 & 153.0 & 475.0 & $0.63 \pm 0.07$ & $<1.9$ & $\mathrm{~h}, \mathrm{l}$ \\
\hline VLAJ123629+621045 & $<15.4$ & 71.6 & $>0.86$ & - & 1 \\
\hline VLAJ123631+620957 & $<26.7$ & 156.0 & $>0.99$ & 1.8 & 1 \\
\hline VLAJ123632+621105 & 21.8 & $<23.0$ & $<0.10$ & $<3.5$ & $\mathrm{~h}$ \\
\hline VLAJ123632+620800 & $<36.9$ & 104.0 & $>0.58$ & 2.2 & 1 \\
\hline VLAJ123633+621006 & $<8.4$ & 48.2 & $>0.98$ & - & 1 \\
\hline VLAJ123634+621435 & 14.0 & $<25.0$ & $<0.32$ & - & $\mathrm{h}$ \\
\hline VLAJ123634+621212 & 56.5 & 211.0 & $0.74 \pm 0.06$ & 1.9 & $\mathrm{~h}, \mathrm{l}$ \\
\hline VLAJ123634+621241 & 52.6 & 198.0 & $0.74 \pm 0.06$ & 2.0 & $\mathrm{~h}, 1$ \\
\hline VLAJ123635+621424 & $<14.3$ & 69.7 & $>0.87$ & 2.1 & 1 \\
\hline VLAJ123637+621135 & 17.5 & $<23.0$ & $<0.20$ & $<3.5$ & $\mathrm{~h}$ \\
\hline VLAJ123637+620852 & $<18.5$ & 79.8 & $>0.82$ & - & 1 \\
\hline VLAJ123638+621117 & 12.0 & $<25.0$ & $<0.41$ & - & $\mathrm{h}$ \\
\hline VLAJ $123640+621010$ & 29.2 & 64.5 & $0.44 \pm 0.15$ & 2.1 & h, 1 \\
\hline VLAJ123640+621833 & $<73.5$ & 307.0 & $>0.80$ & $<2.0$ & 1 \\
\hline VLAJ123642+621142 & 18.6 & 30.0 & $<0.27$ & $<6.0$ & $\mathrm{~h}$ \\
\hline VLAJ123641+620948 & $<21.2$ & 57.8 & $>0.56$ & - & 1 \\
\hline VLAJ123642+621331 & 79.9 & 432.0 & $0.94 \pm 0.06$ & 1.8 & $\mathrm{~h}, \mathrm{l}$ \\
\hline VLAJ123642+621545 & 53.6 & 131.0 & $0.50 \pm 0.07$ & 2.0 & $\mathrm{~h}, \mathrm{l}$ \\
\hline VLAJ123644+621249 & 10.2 & $<23.0$ & $<0.46$ & - & $\mathrm{h}$ \\
\hline VLAJ123644+621133 & 752.0 & 1290.0 & $0.30 \pm 0.05$ & 2.0 & $\mathrm{~h}, \mathrm{l}$ \\
\hline VLAJ123646+621448 & 24.7 & 112.0 & $0.84 \pm 0.12$ & 2.0 & $\mathrm{~h}, \mathrm{l}$ \\
\hline VLAJ123646+621445 & 13.3 & 77.2 & $0.98 \pm 0.15$ & 2.8 & $\mathrm{~h}, \mathrm{l}$ \\
\hline VLAJ123646+621404 & 190.0 & 177.0 & $-0.04 \pm 0.06$ & $<2.0$ & $\mathrm{~h}, \mathrm{l}$ \\
\hline VLAJ123646+621630 & $<17.3$ & 312.0 & $>1.62$ & 2.2 & 1 \\
\hline
\end{tabular}




\begin{tabular}{|c|c|c|c|c|c|}
\hline VLAJ123646+620833 & $<29.6$ & 87.9 & $>0.61$ & - & 1 \\
\hline VLAJ123646+621226 & $<9.5$ & 72.3 & $>1.14$ & 2.9 & 1 \\
\hline VLAJ123649+621312 & 14.0 & 51.0 & $0.72 \pm 0.15$ & $<6$ & $\mathrm{~h}, \mathrm{l}$ \\
\hline VLAJ123649+620737 & 113.0 & 310.0 & $0.56 \pm 0.07$ & $<2.0$ & $\mathrm{~h}, \mathrm{l}$ \\
\hline VLAJ123650+620844 & $<20.6$ & 86.2 & $>0.80$ & - & 1 \\
\hline VLAJ123651+621030 & 26.0 & 98.7 & $0.74 \pm 0.14$ & $<2.1$ & $\mathrm{~h}, 1$ \\
\hline VLAJ123651+621221 & 16.8 & 60.2 & $0.71 \pm 0.12$ & - & $\mathrm{h}, \mathrm{l}$ \\
\hline VLAJ123652+621444 & 185.0 & 148.0 & $-0.12 \pm 0.07$ & 1.9 & $\mathrm{~h}, \mathrm{l}$ \\
\hline VLAJ123653+621139 & 15.1 & 60.0 & $0.77 \pm 0.12$ & - & $\mathrm{h}, \mathrm{l}$ \\
\hline VLAJ123654+621039 & $<8.0$ & 48.2 & $>1.00$ & - & 1 \\
\hline VLAJ123655+621311 & 12.3 & $<23.0$ & $<0.26$ & - & $\mathrm{h}$ \\
\hline VLAJ123656+620917 & $<24.9$ & 67.0 & $>0.55$ & - & 1 \\
\hline VLAJ123655+620808 & $<23.5$ & 107.0 & $>0.85$ & $<2.1$ & 1 \\
\hline VLAJ123656+621207 & $<4.8$ & 45.7 & $>1.32$ & - & 1 \\
\hline VLAJ123656+621302 & $<6.8$ & 60.3 & $>1.22$ & - & 1 \\
\hline VLAJ123658+621455 & 15.3 & $<23.0$ & $<0.20$ & - & $\mathrm{h}$ \\
\hline VLAJ123659+621435 & 11.4 & $<25.0$ & $<0.44$ & - & $\mathrm{h}$ \\
\hline VLAJ123659+621832 & 317.0 & 502.0 & $0.26 \pm 0.07$ & $<2.0$ & $\mathrm{~h}, \mathrm{l}$ \\
\hline VLAJ123700+620909 & 66.7 & 326.0 & $0.89 \pm 0.12$ & $<2.0$ & $\mathrm{~h}, 1$ \\
\hline VLAJ123701+621146 & 29.5 & 97.7 & $0.67 \pm 0.08$ & 2.2 & $\mathrm{~h}, 1$ \\
\hline VLAJ123702+621401 & $<10.0$ & 41.4 & $>0.79$ & - & 1 \\
\hline VLAJ123705+621153 & $<6.0$ & 59.1 & $>1.27$ & - & 1 \\
\hline VLAJ123707+621408 & 29.0 & 48.6 & $0.29 \pm 0.16$ & - & $\mathrm{h}, \mathrm{l}$ \\
\hline VLAJ123707+621121 & $<14.8$ & 64.0 & $>0.82$ & - & 1 \\
\hline VLAJ123708+621056 & 26.4 & 49.1 & $0.35 \pm 0.17$ & - & $\mathrm{h}, \mathrm{l}$ \\
\hline VLAJ123709+621246 & 19.5 & $<23.0$ & $<0.10$ & $<3.5$ & $\mathrm{~h}$ \\
\hline VLAJ123709+620837 & 122.0 & 64.8 & $-0.35 \pm 0.11$ & - & $\mathrm{h}, \mathrm{l}$ \\
\hline VLAJ123711+621331 & 31.1 & 108.0 & $0.69 \pm 0.13$ & $<2.1$ & $\mathrm{~h}, 1$ \\
\hline VLAJ123711+621325 & $<13.7$ & 109.0 & $>1.16$ & - & 1 \\
\hline VLAJ123713+621826 & $<101.4$ & 527.5 & $>0.92$ & $<1.9$ & 1 \\
\hline VLAJ123714+620823 & 1060.0 & 1380.0 & $0.15 \pm 0.08$ & $<1.9$ & $\mathrm{~h}, 1$ \\
\hline VLAJ123716+621512 & 85.8 & 180.0 & $0.41 \pm 0.09$ & $<2.0$ & $\mathrm{~h}, \mathrm{l}$ \\
\hline VLAJ123716+621733 & $<77.2$ & 302.0 & $>0.76$ & $<2.0$ & 1 \\
\hline VLAJ123717+620827 & $<15.7$ & 62.6 & $>0.77$ & - & 1 \\
\hline VLAJ123721+621130 & 630.0 & 383.0 & $-0.28 \pm 0.06$ & 1.9 & $\mathrm{~h}, 1$ \\
\hline VLAJ123721+621346 & $<12.4$ & 40.7 & $>0.66$ & - & 1 \\
\hline
\end{tabular}


$\begin{array}{llllll}\text { VLAJ123725+621128 } & 530.0 & 5960.0 & 1.35 \pm 0.06 & 0.8 & \text { h, } 1\end{array}$

\begin{tabular}{lllllll} 
VLAJ123730+621258 & $<27.1$ & 112.2 & $>0.79$ & $<2.1$ & 1 \\
\hline
\end{tabular}




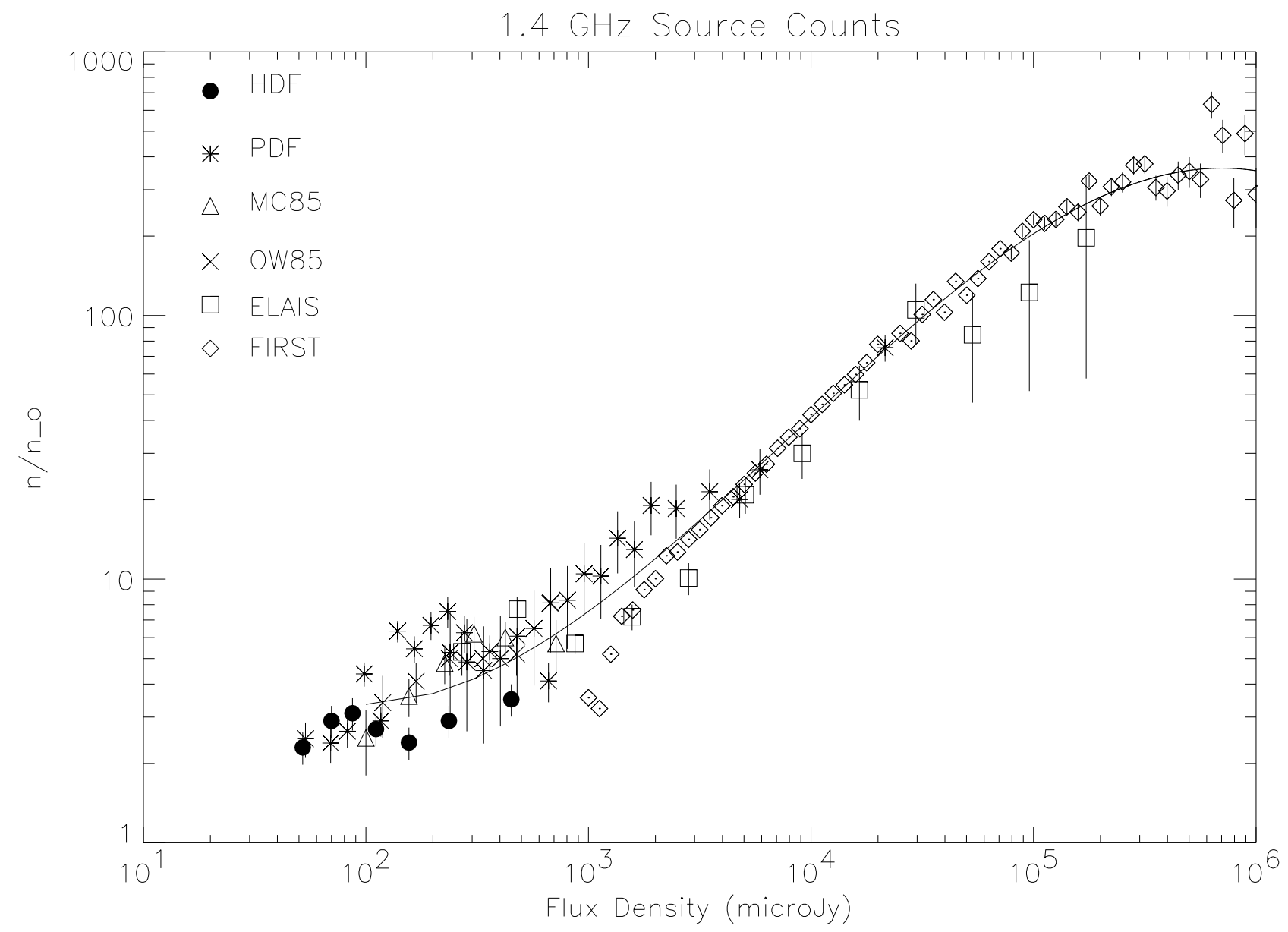




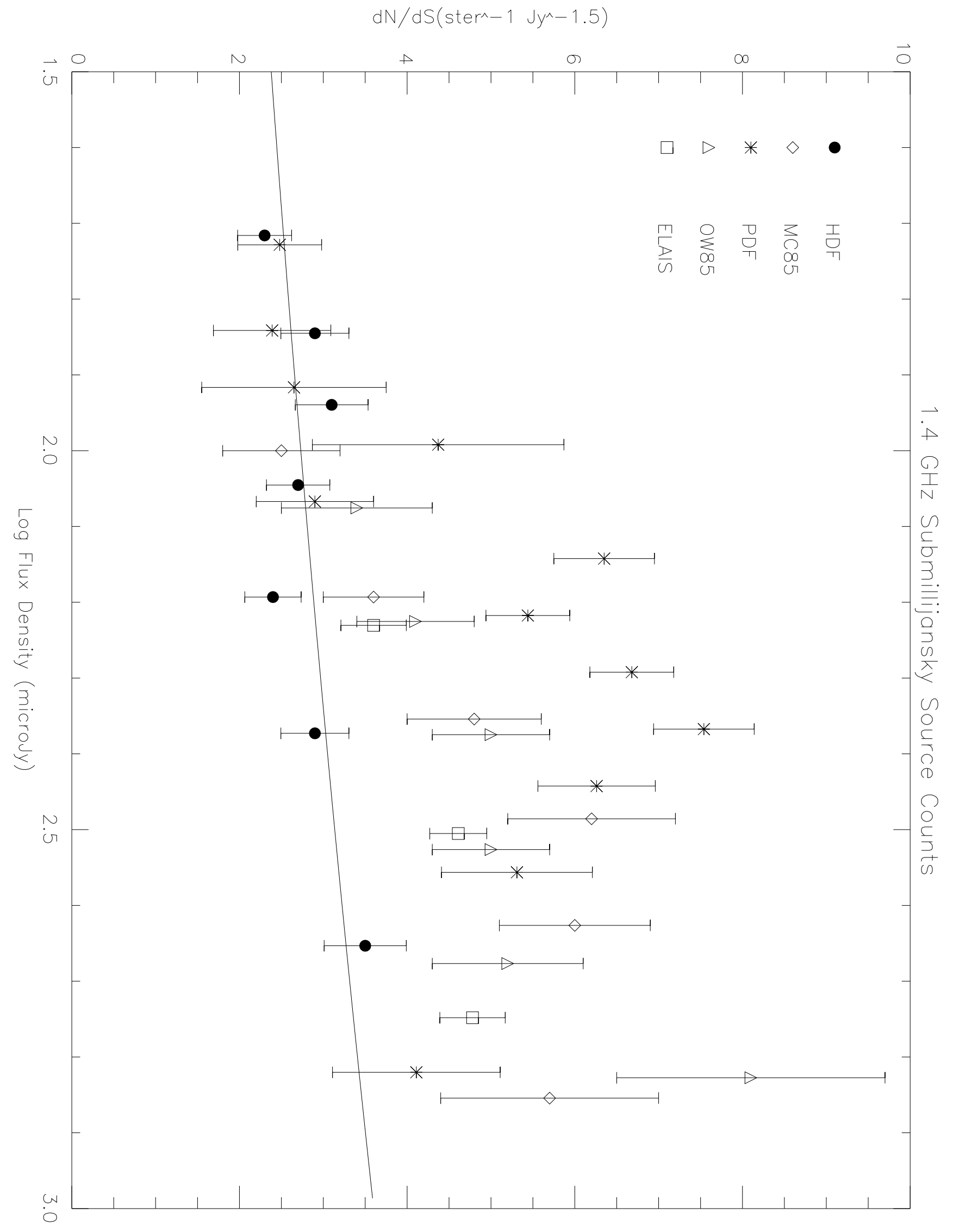




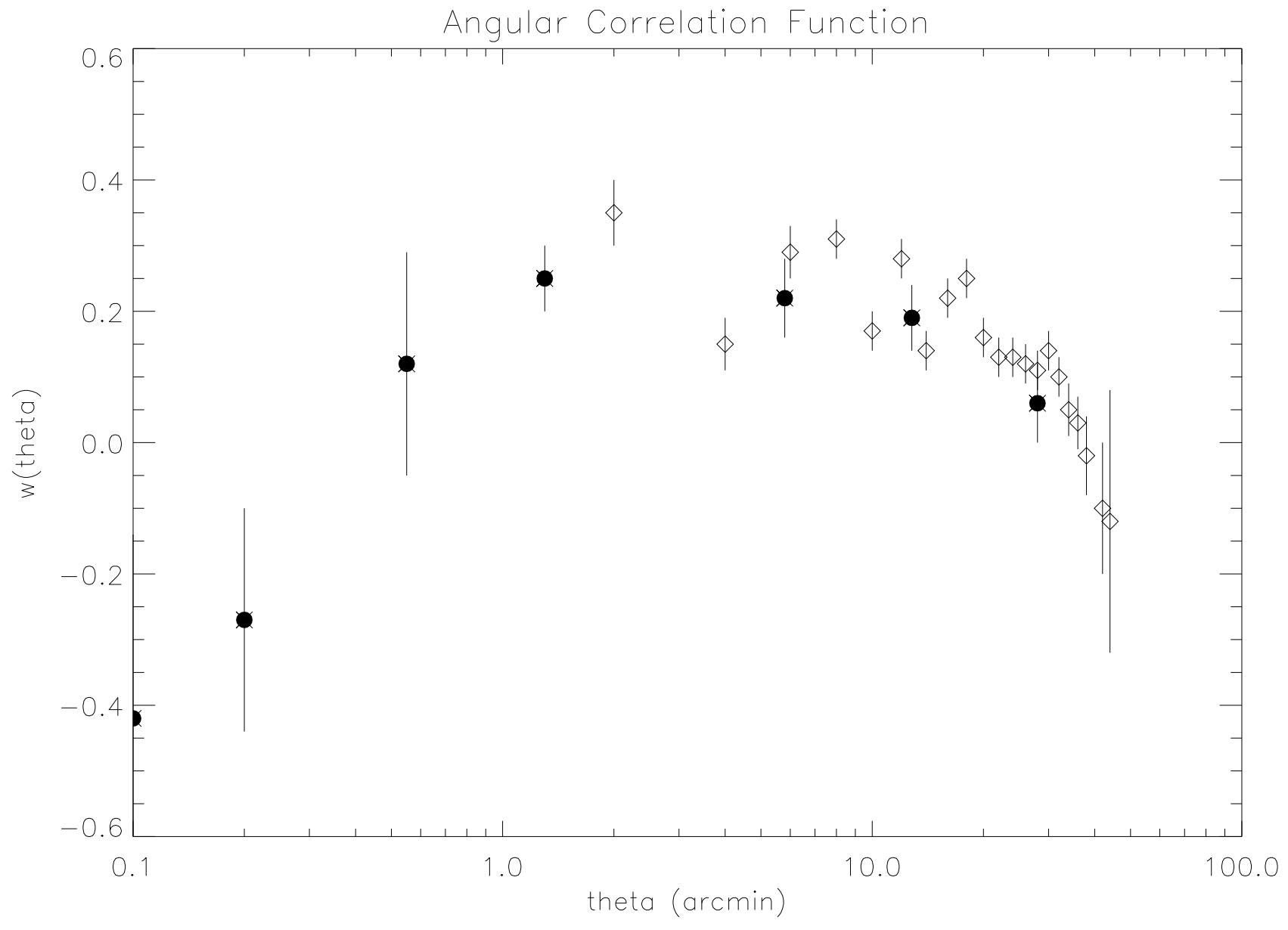




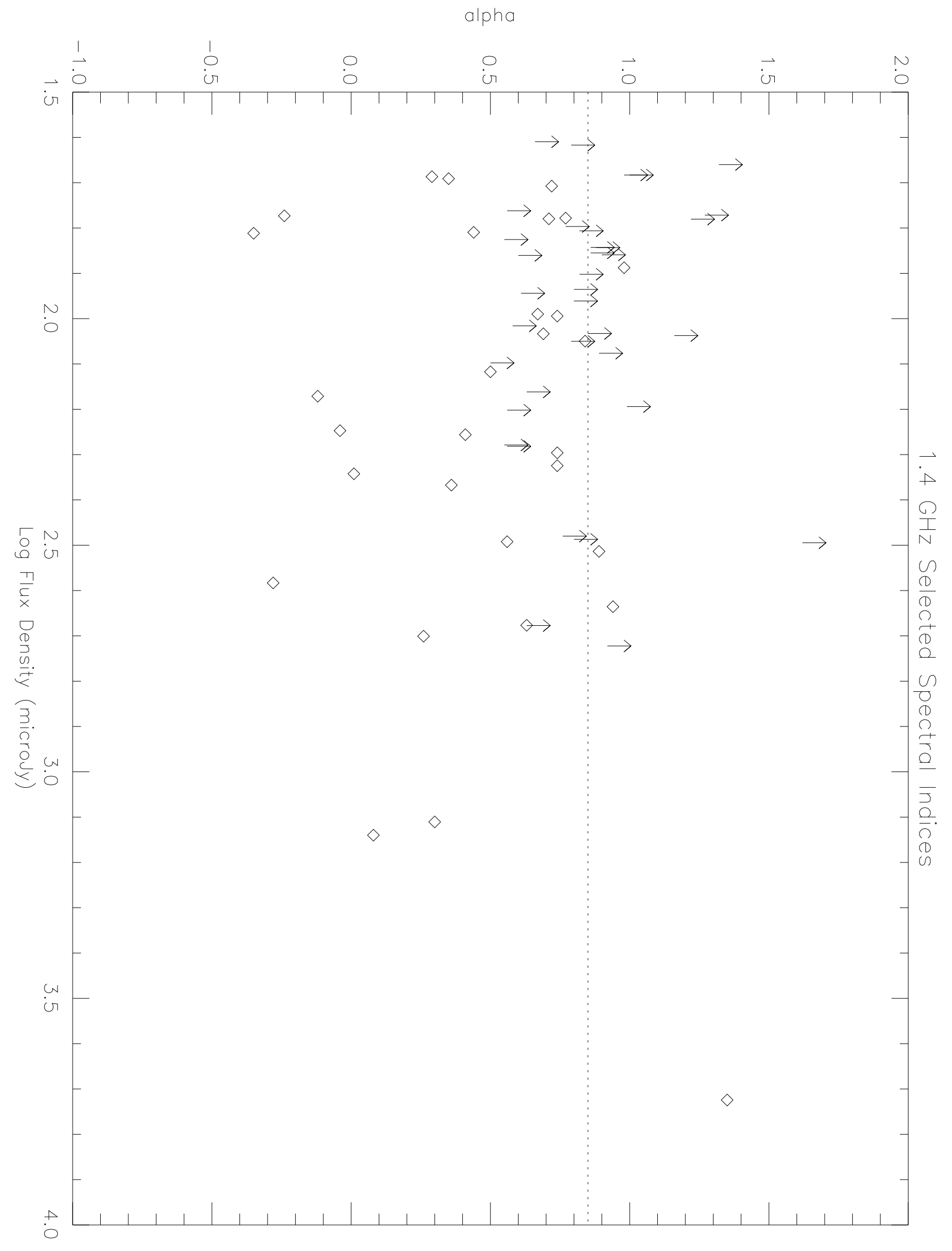




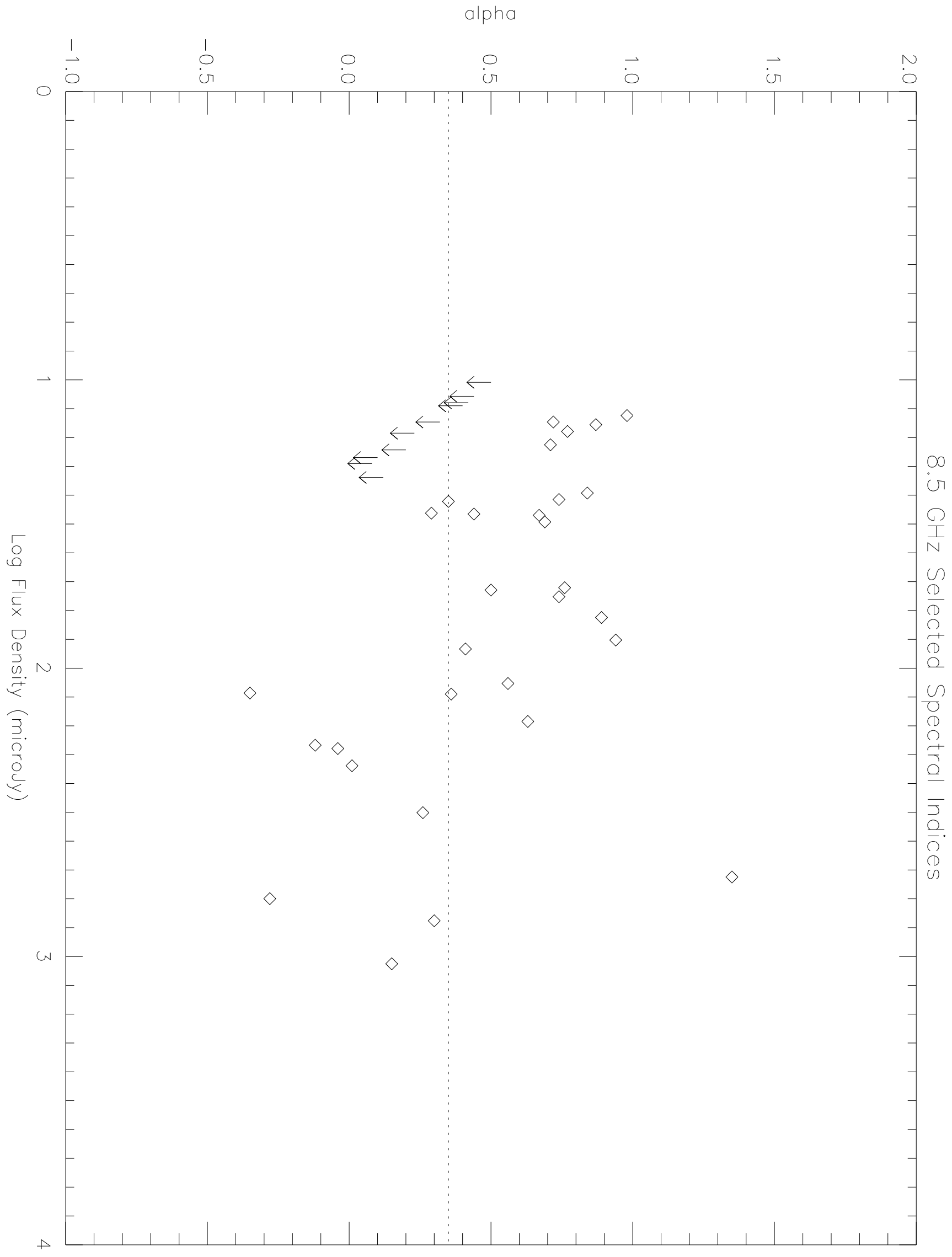


Table 1

VLA/Hubble Deep Field Imaging Parameters

\begin{tabular}{rrrrrrrrr}
\hline \hline $\begin{array}{r}\text { Freq. } \\
(\mathrm{GHz})\end{array}$ & $\begin{array}{r}\text { Res. } \\
(\mathrm{arcsec})\end{array}$ & $\begin{array}{r}\text { Size } \\
(\text { Pixels })\end{array}$ & $\begin{array}{r}\text { Pixel } \\
(\mathrm{arcsec})\end{array}$ & $\begin{array}{r}\text { Clean } \\
\text { Comp }\end{array}$ & $\begin{array}{r}\text { Pixel } \\
\operatorname{Max}(\mu \mathrm{Jy})\end{array}$ & $\begin{array}{r}\text { Pixel } \\
\operatorname{Min}(\mu \mathrm{Jy})\end{array}$ & $\begin{array}{r}\text { rms } \\
\mu \mathrm{Jy} / \mathrm{beam}\end{array}$ & $\begin{array}{r}\text { Comp } \\
\mu \mathrm{Jy}\end{array}$ \\
\hline \hline 1.4 & 1.2 & 8192 & 0.4 & 160000 & 30000 & -60 & 9.1 & - \\
1.4 & 2.0 & 8192 & 0.4 & 160000 & 35000 & -67 & 7.5 & 40.0 \\
1.4 & 3.5 & 4096 & 0.8 & 80000 & 51000 & -71 & 9.7 & 50.0 \\
1.4 & 6.0 & 2048 & 1.6 & 16000 & 63000 & -101 & 14.7 & 75 \\
8.5 & 3.5 & 2048 & 0.4 & 20000 & 630 & -8.5 & 1.6 & 8.0 \\
\hline \hline
\end{tabular}

